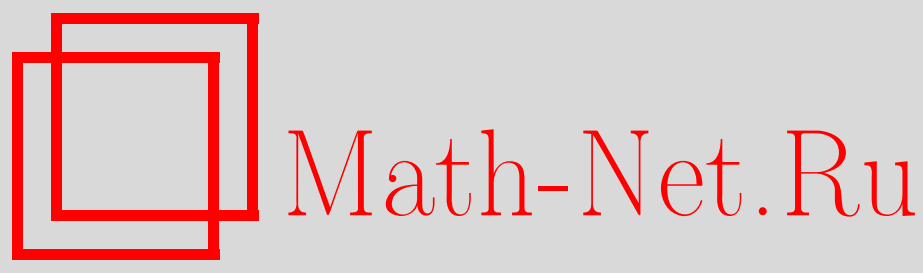

Ю. А. Дабагян, Аналитическое описание статистики спектров квантовых графов, ТМФ, 2008, том 156, номер 1, 38-66

DOI: https://doi.org/10.4213/tmf6229

Использование Общероссийского математического портала Math-Net.Ru подразумевает, что вы прочитали и согласны с пользовательским соглашением http://www . mathnet.ru/rus/agreement

Параметры загрузки:

IP : 54.205 .225 .156

26 апреля 2023 г., 13:08:37

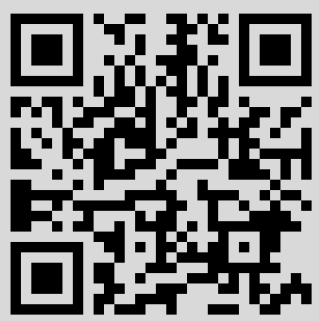




\title{
АНАЛИТИЧЕСКОЕ ОПИСАНИЕ СТАТИСТИКИ СПЕКТРОВ КВАНТОВЫХ ГРАФОВ
}

\begin{abstract}
Обсуждается получение точных и приближенных распределений для различных статистических характеристик спектров квантовых графов, полученных на основе ранее найденных точных решений спектральной задачи. Указывается связь возникающих спектральных разложений с теорией слабо зависимых случайных величин, а также отмечена возникающая связь между известными предельными теоремами для тригонометрических сумм и универсальными статистическими свойствами спектров квантово-хаотических систем.
\end{abstract}

Ключевые слова: квантовый хаос, спектральная теория, универсальные распределения, почти независимые случайные величины, аналитические методы.

\section{1. ВВЕДЕНИЕ}

Рассмотрим точечную классическую частицу, движущуюся вдоль ребер произвольного конечного графа $G$, состоящего из $N_{\mathrm{P}}$ ребер и $N_{\mathrm{B}}$ вершин. В простейшем случае можно считать, что частица движется вдоль ребер графа свободно, а после рассеяния на очередной вершине $V_{i}$ она может, с различными вероятностями, продолжить движение вдоль любого из ребер $P_{i j}$, соединяющихся в $V_{i}$. Траектории частицы в такой системе представляют собой различные последовательности сочлененных ребер графа. В результате каждого рассеяния на вершинах информация о предыстории движения частицы полностью теряется. Следовательно, динамика такой частицы необратима и представляет собой марковский процесс.

Задаваемая таким образом стохастическая динамика имеет несколько важных черт, характерных для общих (в том числе динамических) неинтегрируемых систем [1]-[4]. Например, в данной модели присутствуют свойства изолированности и разбегания траекторий [5]-[7], поскольку любые две траектории частицы на графе или полностью совпадают, или после рассеяния на очередной вершине продолжаются вдоль различных последовательностей ребер. Из-за неоднозначности рассеяния на вершинах число возможных орбит, в частности периодических орбит, возрастает экспоненциально со временем или с числом рассеяний на вершинах. При этом показатель экспоненциального роста (топологическая энтропия) такой системы зависит

* Department of Physics, University of California, San Francisco, California, USA. E-mail: yura@phy.ucsf.edu 
только от топологии графа, но не от значений вероятностей рассеяния на вершинах. Таким образом, несмотря на то что показатель Ляпунова нельзя определить локально для каждого отдельного акта "разбегания" (расщепления орбит на вершинах графа), глобальная топологическая энтропия хорошо определена и выступает в роли показателя хаотичности.

Для справедливости обсуждаемых ниже свойств графов существенным является предположение, что частица, попавшая на ребро $P_{i j}$ через вершину $V_{i}$, обязательно достигает вершины $V_{j}$, где ее дальнейшее поведение целиком определяется заранее заданными вероятностями рассеяния.

Ребра графа могут быть оснащены потенциалами $U_{i j}(x, k)$, зависящими от координаты $x$ и, вообще говоря, от импульса $k$. В дальнейшем мы будем подразумевать наличие постоянных скейлинговых потенциалов $U_{i j}(x, k)=\lambda_{i j} k^{2}$, обсуждавшихся ранее в [8]-[16].

Поскольку и классическое, и квантовое описания квантовых графов сравнительно легко доступны, представляет интерес изучение этих систем в контексте теории "квантового хаоса" [6], [7], [17] - точное и статистическое описание спектра, нахождение плотности состояний, проверка различных феноменологических универсальных закономерностей и т.д.

Обсуждение квантовых графов в контексте теории квантового хаоса было начато в серии публикаций [2]-[4] (см. также обзоры [18], [19]), где было численно показано, что статистическое поведение различных спектральных характеристик соответствующего оператора Шредингера, например распределение вероятностей расстояний между ближайшими уровнями импульса $s=k_{n}-k_{n-1}$, хорошо соответствует предсказаниям теории случайных матриц [20]-[22], как это обычно имеет место для систем, являющихся хаотичными в классическом пределе (см. рис. 1).

Также было показано, что определенные глобальные спектральные характеристики, такие как спектральная лестница

$$
N(k) \equiv \sum_{j=1}^{\infty} \Theta\left(k-k_{j}\right)
$$

(число уровней $k_{n}$, лежащих ниже некоторого фиксированного значения $k$ ) и соответствующая ей квантово-механическая плотность состояний

$$
\rho(k) \equiv \sum_{j=1}^{\infty} \delta\left(k-k_{j}\right),
$$

могут быть представлены в виде точных разложений по классическим периодическим орбитам. Например,

$$
N(k)=\bar{N}(k)+\frac{1}{\pi} \operatorname{Im} \sum_{p} A_{p} e^{i L_{p}},
$$

где $\bar{N}(k)$ - среднее число уровней на интервале $[0, k], L_{p}$ - действие периодической траектории с индексом $p$, а $A_{p}$ - весовой множитель [4], [8], [23]. 


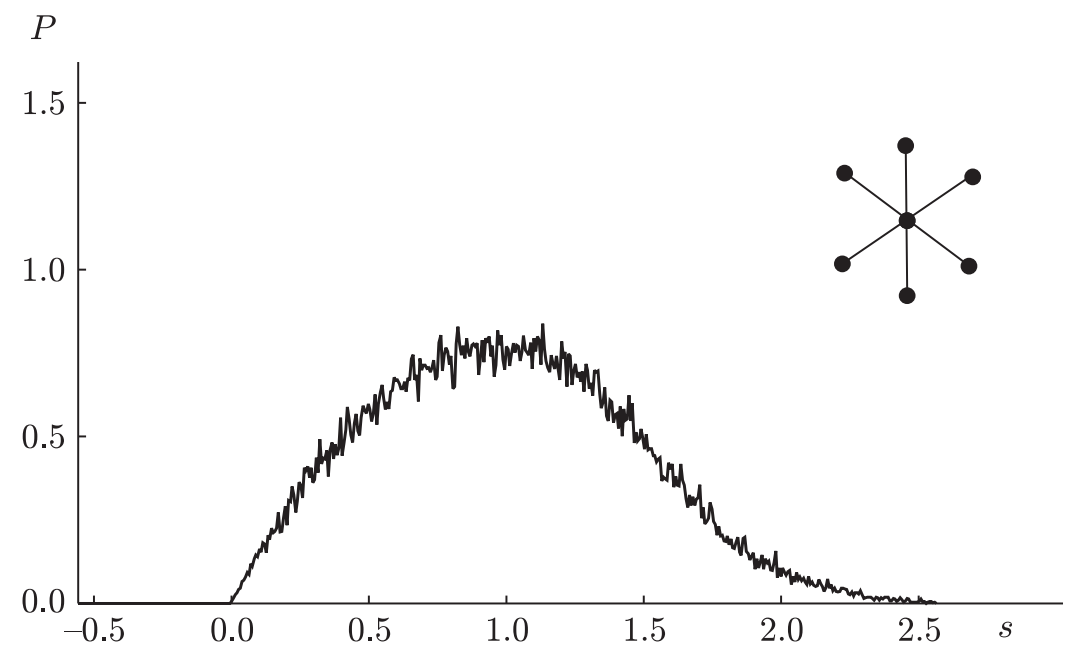

Рис. 1. Гистограмма 80000 значений $s=k_{n}-k_{n-1}$ для случая неоснащенного графа с шестью ребрами, исходящими из одной точки (шестивершинной гидры). Горизонтальная шкала задана в единицах $\pi / L_{0}$, где $L_{0}$ - сумма оптических длин ребер графа. Общие черты распределения, например явления отталкивания уровней, $P(0)=0$, и экспоненциальный вид хвоста $P(s)$ характерны для общих квантово-хаотических систем [20]-[22].

Разложения вида $(3)$ для $N(k)$ и $\rho(k)$ являются одними из основных результатов квазиклассической теории для классически неинтегрируемых систем [6]-[8], [17], [21], [23]-[26]. При этом важно отметить, что нахождение таких разложений не эквивалентно окончательному решению спектральной задачи в виде формул для $k_{n}$, как это имеет место в теории квантования Бора-Зоммерфельда [27], [28] или теории Эйнштейна-Бриллюэна-Келлера [29]-[31] для интегрируемых систем, поскольку разложения (3) описывают не отдельные уровни, а весь спектр целиком. Для окончательного нахождения значений квантовых уровней энергии с помощью плотности состояний необходима дополнительная информация о том, где именно расположены отдельные дельта-пики в (2). Если можно показать, что дельта-пик в $\rho(k)$, соответствующий уровню $k_{n}$, расположен между точками $\hat{k}_{n-1}$ и $\hat{k}_{n}$, то значение $k_{n}$ можно найти по формуле

$$
k_{n}=\int_{\hat{k}_{n-1}}^{\hat{k}_{n}} k \rho(k) d k .
$$

Априори такая возможность отсутствует, поскольку отсутствует информация о расположении отдельных дельта-пиков в (2) и тем более отсутствует глобальная информация, позволяющая находить точки $\hat{k}_{n}$ систематически, в виде глобально определенной функции от $n$ (поскольку в хаотической системе энергия является единственным интегралом движения, то для нее существует единственное квантовое число $\left.n=N\left(k_{n}\right)\right)$. Тем не менее, как было показано в [13]-[15], квантовые 
графы представляют собой примеры систем, для которых такой набор разделяющих точек $k_{n}, n=1,2, \ldots$, можно построить явно, исходя из свойств спектрального детерминанта, и тем самым предъявить алгоритм для решения спектральной задачи в рамках квазиклассической теории.

Дальнейшее изложение посвящено обсуждению точного решения спектральной задачи, предложенного в [13]--[15], и выводу статистического описания спектральных характеристик, возникающего на его основе.

\section{2. СПЕКТРАЛЬНОЕ УРАВНЕНИЕ}

Наиболее наглядным и естественным с точки зрения квазиклассической теории методом построения спектрального детерминанта является метод, предложенный Богомольным [32], [33]. Согласно этому методу спектральный детерминант получается из условий совместности отображения волновой функции на сечении Пуанкаре в конфигурационном пространстве системы и имеет вид

$$
\Delta(E)=\operatorname{det}(1-T(E)),
$$

где под $T(E)$ подразумевается оператор

$$
T\left(q, q^{\prime}, E\right)=\frac{1}{(2 \pi \hbar)^{1 / 2}} \sum_{p}\left|\frac{\partial^{2} S_{p}\left(q, q^{\prime}, E\right)}{\partial q \partial q^{\prime}}\right|^{1 / 2} \exp \left[\frac{i}{\hbar} S_{p}\left(q, q^{\prime}, E\right)-i \frac{\pi \nu_{p}}{2}\right] .
$$

Здесь $S_{p}\left(q, q^{\prime}, E\right)$ обозначает действие траектории $p$, соединяющей точки $q$ и $q^{\prime}$ фазового пространства на поверхности сечения Пуанкаре (ПСП), $\nu_{p}$ - индекс Маслова. Суммирование производится по всем траекториям с энергией $E$, ведущим непосредственно (т.е. без промежуточных пересечений с ПСП) из $q$ в $q^{\prime}$, с одинаковым направлением импульса. Для нахождения уровней энергии, заключенных в интервале $[0, E]$, в пределе $\hbar \rightarrow 0$ используется конечномерная матрица размера $N(E) \times N(E)$, определяющегося фазовым объемом ПСП для данной энергии, $N(E)=\operatorname{Vol}(\Sigma(E)) /(2 \pi \hbar)^{d-1}$.

В случае, когда конфигурационное пространство представляет собой граф, в качестве ПСП можно выбрать множество вершин графа $V_{1}, \ldots, V_{N_{\mathrm{B}}}$, и тогда "сечение" будет представлять собой дискретный набор точек, соединенных между собой ребрами графа (рис. 2). В результате размер матрицы $T(E)$ всегда конечен и ее структура определяется топологией графа. Очевидно, что траектория, соединяющая точки ПСП $q$ и $q^{\prime}$, существует, если имеется ребро графа, соединяющее соответствующие вершины. Явный вид элементов матрицы переноса $T(k)$ можно получить, прослеживая движение частицы вдоль ребер, учитывая при этом изменения амплитуды волновой функции из-за рассеяний и отражений, а также различая "вхождения" и "выхождения" из ПСП. Можно показать, что если частица выходит из вершины $V_{i}$, достигает вершины $V_{j}$, пройдя вдоль ребра $L_{i j}$, рассеивается на ней и снова выходит из ПСП, продолжая движение в направлении вершины $V_{k}$, то такая последовательность событий описывается произведением амплитуды $e^{i L_{i j}}$ на коэффициент рассеяния $t_{j k}$, что и задает величину соответствующего матричного элемента $T(k)$. 


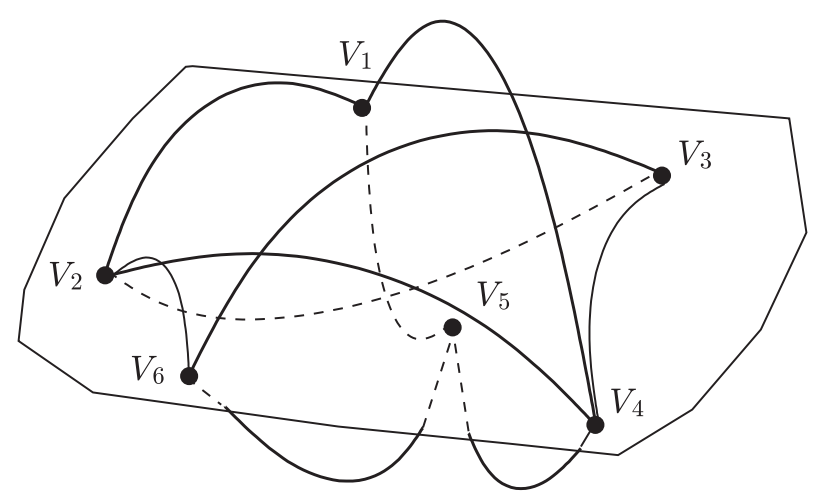

Рис. 2. Поверхность сечения Пуанкаре в конфигурационном пространстве графа. Для наглядности вершины расположены в двумерной плоскости.

Таким образом, размер $2 N_{\mathrm{P}} \times 2 N_{\mathrm{P}}$ матрицы $T(E)$ определяется количеством ребер графа. Например, для случая двухреберной гидры с длинами ребер $l_{1}$ и $l_{2}$ [8]-[15] ПСП состоит из трех вершин графа $V_{1}, V_{2}, V_{3}$. С учетом направлений пересечений орбит с ПСП и знаков коэффициентов рассеяний [8] согласно правилам построения оператора $T(k)$ получаем

$$
\left(\begin{array}{l|llll} 
& V_{1} \rightarrow V_{2} & V_{2} \rightarrow V_{1} & V_{2} \rightarrow V_{3} & V_{3} \rightarrow V_{2} \\
\hline V_{1} \rightarrow V_{2} & 0 & r e^{i k l_{1}} & t e^{i k l_{1}} & 0 \\
V_{2} \rightarrow V_{1} & -e^{i k l_{1}} & 0 & 0 & 0 \\
V_{2} \rightarrow V_{3} & 0 & 0 & 0 & -e^{i k l_{2}} \\
V_{3} \rightarrow V_{2} & 0 & t e^{i k l_{2}} & -r e^{i k l_{2}} & 0
\end{array}\right) .
$$

Получающийся спектральный детерминант записывается в виде

$$
\Delta(k)=r e^{2 i k l_{1}}-r e^{2 i k l_{2}}-e^{2 i k\left(l_{1}+l_{2}\right)}+1 .
$$

После выделения комплексной фазы, задаваемой полной длиной ребер графа, $\Theta(k)=$ $k\left(l_{1}+l_{2}\right)=k L_{0}$, получаем спектральное уравнение

$$
|\Delta(k)|=\sin \left(l_{1}+l_{2}\right)-r \sin \left(l_{1}-l_{2}\right)=0 .
$$

В работах [2], [12] было показано, что выражение (5) можно также получить из условия совместности граничных условий для волновой функции, решив вспомогательную квантовую задачу рассеяния на графе. В контексте такого решения матрица $T(k)$ имеет смысл унитарной матрицы рассеяния на графе.

Из вида спектрального детерминанта (5) и формы оператора $T$ легко выводятся основные формулы теории периодических орбит, например квазиклассическое разложение для плотности корней спектрального уравнения (формула Гутцвиллера) или разложение для спектральной лестницы (1)

$$
N(k)=\bar{N}(k)+\operatorname{Im} \sum_{n=1}^{\infty} \frac{1}{n} \operatorname{Tr}\left(T^{n}(k)\right) .
$$


Среднее количество уровней $\bar{N}$ определяется фазой $\Theta(k)$ спектрального детерминанта [9]-[12], [32], [33].

Как и в приведенном выше примере, в общем случае спектральный детерминант квантовых графов представляет собой конечную экспоненциальную сумму,

$$
\Delta(k)=1+\sum_{i=1}^{N_{\Gamma}} a_{i} e^{i k L_{(i)}}
$$

порядок $N_{\Gamma}$ которой определяется топологией графа, а $L_{(i)}$ представляют собой различные алгебраические комбинации оптических длин ребер $l_{1}, \ldots, l_{N_{\mathrm{P}}}[11],[12]$. После выделения комплексной фазы спектральное уравнение $|\Delta(k)|=0$ может быть записано в виде

$$
\cos \left(k L_{0}+\pi \gamma_{0}\right)=-\sum_{i=1}^{N_{\Gamma}} a_{i} \cos \left(L_{(i)} k+\gamma_{i}\right),
$$

где максимальная частота $L_{0}$ задается суммой оптических длин ребер графа [9]-[15]. Эта форма записи позволяет произвести классификацию спектральных детерминантов [13]-[15] в соответствии с величиной в правой части уравнения (12), обозначаемой в [12] как $\Phi(k)$. Можно показать, что если функция $\Phi(k)$ удовлетворяет условию

$$
f \equiv \max _{k}|\Phi(k)|<1
$$

для всех значений $k \in \mathbb{R}$, то спектральное уравнение можно формально обратить,

$$
k_{n}=\frac{\pi}{L_{0}}\left(n+\frac{1}{2}\right) \pm \frac{1}{L_{0}} \arccos \Phi\left(k_{n}\right),
$$

сохраняя однозначность решения (12). Системы, для которых условие (13) выполнено, назывались регулярными системами в [9]-[12], где также было показано, что в случае выполнения условия (13) значения $k_{n}$ отделяются друг от друга периодической последовательностью (см. рис. 3),

$$
\hat{k}_{n}=\frac{\pi}{L_{0}}\left(n+\frac{1}{2}\right) .
$$

Достаточным условием выполнения (13) является неравенство

$$
\alpha \equiv \sum_{i=1}^{N_{\Gamma}}\left|a_{i}\right|<1
$$

где $a_{i}$ - коэффициенты в разложении (11). В терминах свойств функций $N(k)$ и $\bar{N}(k)$ условие (13) означает, что вейлевское среднее $\bar{N}(k)$, представляющее собой прямую с наклоном $L_{0} / \pi$, является "сквозным", т.е. пересекает каждую ступеньку спектральной лестницы в точках (15).

В общем случае условие (13) не выполняется, поскольку, вообще говоря, геометрически невозможно “прошить” каждую ступеньку спектральной лестницы общего 


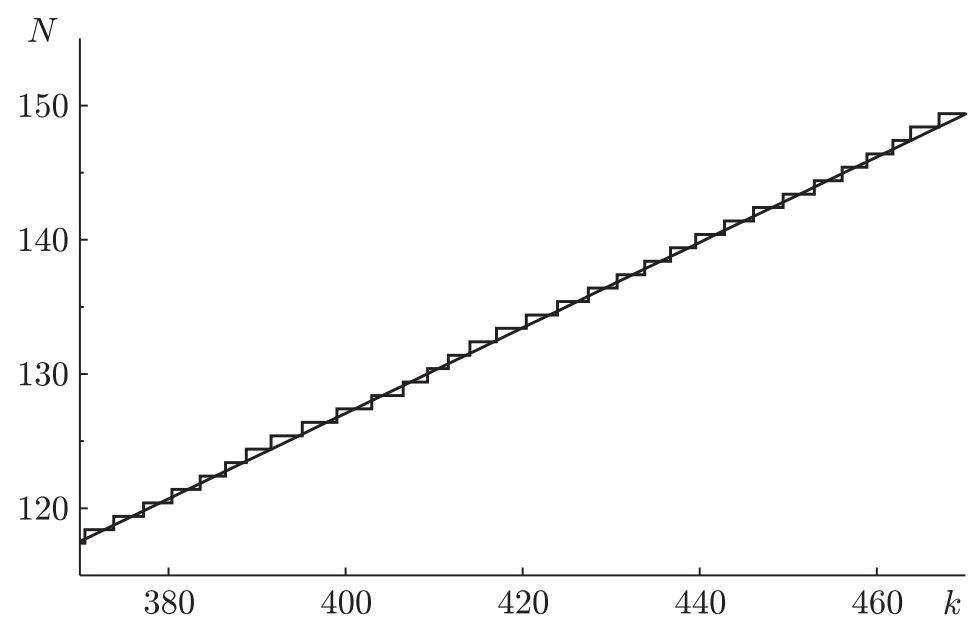

Рис. 3. Вид спектральной лестницы для регулярной последовательности, пересекаемой “сквозным” вейлевским средним $\bar{N}(k)$. Такой вид имеет, например, график $N(k)$ корней уравнения (9) [9]-[12].

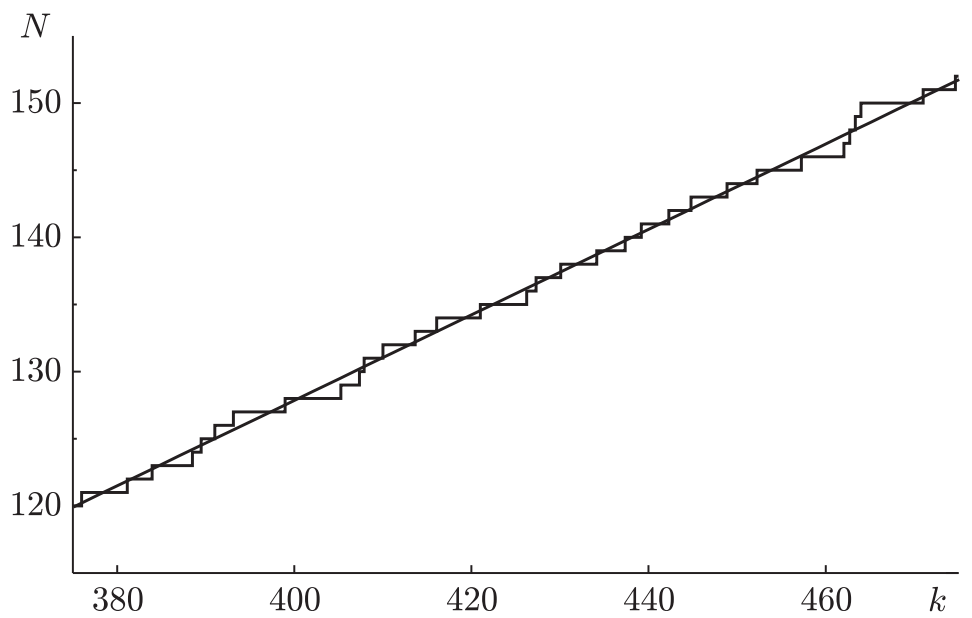

Рис. 4. Пример спектральной лестницы, полученной для спектра нерегулярного шестивершинного квантового графа того же объема $L_{0}$ и на том же интервале $k_{n}$, что и на рис. 3 , пересекаемой монотонной кривой $f(k)$. Распределение величин $s=k_{n}-k_{n-1}$ для этой системы показано на рис. 1 . 
положения одной единственной прямой. В частности, это нельзя сделать прямой, задаваемой вейлевским средним (рис. 4). Для такой операции потребуется найти монотонную кривую $f(k)$, которая будет следовать за спектральной лестницей $N(k)$, пересекая каждую ступень ровно один раз. Порождаемые таким образом разделительные точки $\hat{k}_{n}, f\left(\hat{k}_{n}\right)=N\left(\hat{k}_{n}\right)$, будут задаваться функцией, более сложной, чем $(15), \hat{k}_{n}=f^{-1}(n)$. В частности, получающиеся точки $\hat{k}_{n}$ уже не будут расположены периодически.

Известно, однако, что задача о нахождении такой кривой, способной связать спектральные значения с последовательностью квантового числа (натуральной последовательностью (15)), в общем виде крайне сложна [34]. Регулярные квантовые графы являются единственным известным автору примером классически неинтегрируемой системы, для которой такая связь имеется в явном виде.

Тем не менее последовательность (15) может быть использована опосредованно для выявления организации спектра. Как показано в [13]-[15], для этого можно воспользоваться вспомогательной системой сплетенных последовательностей $k_{n}^{(j)}$, $j=0, \ldots, r+1$, которая включает в себя как последовательность $\hat{k}_{n}=k_{n}^{(r+1)}$, задаваемую формулой (15), так и изначальную физическую последовательность $k_{n}=k_{n}^{(0)}$. Сплетение последовательностей означает, что между каждыми двумя ближайшими точками $k_{n}^{(j)}$ и $k_{n+1}^{(j)}$ существует ровно одна точка $(j-1)$-й последовательности $k_{n}^{(j-1)}$,

$$
k_{n}^{(j)}<k_{n}^{(j-1)}<k_{n+1}^{(j)} .
$$

Можно также показать, что если первоначальная (развернутая) спектральная последовательность имеет вид

$$
k_{n}^{(0)}=\frac{\pi}{L_{0}}\left(n+\delta_{n}^{(0)}\right)
$$

где $\bar{k}_{n}^{(0)}=\pi n / L_{0}$ задает среднее значение $k_{n}^{(0)}$, а $\delta_{n}^{(0)}$ описывает флуктуации с нулевым средним, то и дальнейшие последовательности $k_{n}^{(j)}$, удовлетворяющие условию (17), тоже будут обладать этим свойством. При этом каждую очередную последовательность $k_{n}^{(j)}$ можно подбирать более упорядоченной, стараясь уменьшить размер флуктуаций в

$$
k_{n}^{(j)}=\frac{\pi}{L_{0}}\left(n+\delta_{n}^{(j)}\right)
$$

с ростом $j$, пока, наконец, не окажется возможным использовать периодическую последовательность (15) в качестве разделяющей [13]-[15].

Условие (17) и идею последовательного упорядочивания можно проиллюстрировать на примере поведения спектральных лестниц для каждой из последовательностей $k_{n}^{(j)}$ (рис. 5), которые последовательно сплетаются друг с другом, пока, наконец, последняя спектральная лестница не оказывается "сквозной". Очевидно, что решение тем эффективнее, чем быстрее удается вплести периодическую последовательность в последовательность $k_{n}^{(0)}$. Глобально минимизировать отклонение последовательности

$$
k_{n}^{(j)}=\alpha_{n}^{(j-1)} k_{n+1}^{(j-1)}+\left(1-\alpha_{n}^{(j-1)}\right) k_{n}^{(j-1)},
$$




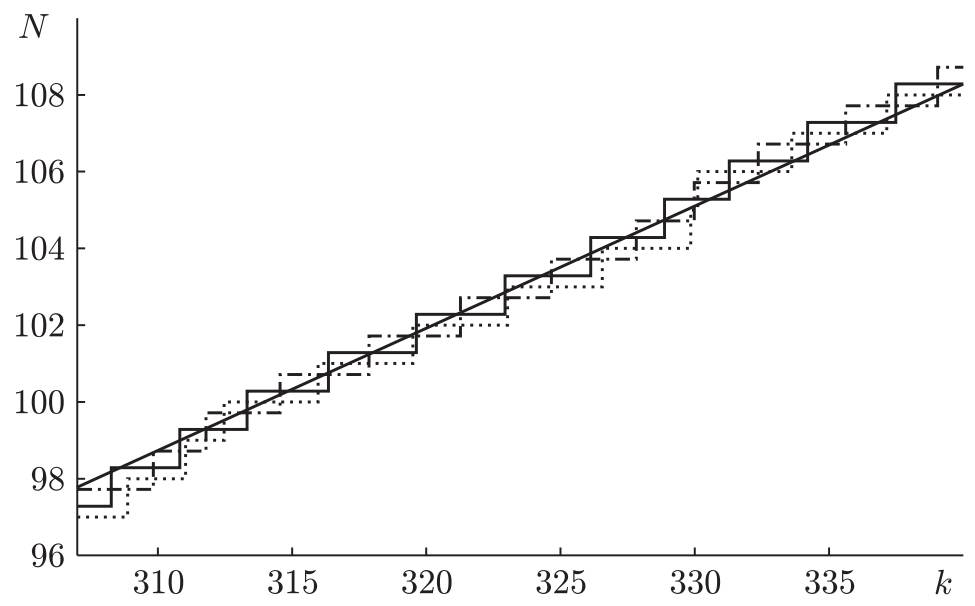

Рис. 5. Сплетение спектральных лестниц $N^{(j)}(k)$ для последовательностей $k_{n}^{(j)}$. Каждая ступенька $N^{(j)}(k)$ пересечена вертикальным отрезком лестницы $N^{(j+1)}(k)$.

где $0<\alpha_{n}^{(j-1)}<1$, вписанной в последовательность точек $k_{n}^{(j-1)}$, от периодической можно, например, из условия минимальности функционала

$$
F=\sum_{n}\left(\left(k_{n}^{(j)}-k_{n-1}^{(j)}\right)-T\right)^{2}=\sum_{n}\left(\left(\alpha_{n}^{(j-1)} s_{n}^{(j-1)}+\left(1-\alpha_{n-1}^{(j-1)}\right) s_{n-1}^{(j-1)}\right)-T\right)^{2},
$$

где

$$
s_{n}^{(j-1)}=\frac{\pi}{L_{0}}\left(1+\delta_{n+1}^{(j-1)}-\delta_{n}^{(j-1)}\right), \quad T=\frac{\pi}{L_{0}} .
$$

Варьируя $F$ относительно параметров $\alpha_{n}^{(j-1)}$ при выполнении условий $0<\alpha_{n}^{(j-1)}<1$ и $k_{n}^{(j)}>k_{n-1}^{(j)}$, получаем для $0<\alpha_{n}^{(j-1)}<1$

$$
\alpha_{n}^{(j-1)}=\frac{\pi}{L_{0}} \frac{1}{s_{n}^{(j)}}\left(\delta_{0}^{(j)}-\delta_{n}^{(j)}\right)
$$

и $\alpha_{n}^{(j-1)}=0$ или $\alpha_{n}^{(j-1)}=1$, если результат $(22)$ выходит за пределы интервала $[0,1]$ соответственно слева или справа.

В разных случаях можно использовать и другие семейства значений коэффициентов $0<\alpha_{n}^{(j-1)}<1$. Например, для простой оценки величины нерегулярности спектра можно использовать разделяющие последовательности, порождаемые $\alpha_{n}^{(j-1)}=1 / 2$,

$$
k_{n}^{(j)}=\frac{\pi}{L_{0}}\left(n+\frac{\delta_{n}^{(j-1)}+\delta_{n+1}^{(j-1)}}{2}\right),
$$

рассматривавшиеся в работе [35]. В [13]-[15] было также показано, что в силу определенных аналитических свойств экспоненциальной суммы вида (11) [15], [36], [37] в качестве $k_{n}^{(j)}$ можно также выбрать последовательность нулей $j$-й производной 

спектрального уравнения

$$
\cos \left(L_{0} k_{n}^{(j)}+\pi \gamma_{0}+\frac{\pi j}{2}\right)+\sum_{i=1}^{N_{\Gamma}} a_{i}\left(\frac{L_{i}}{L_{0}}\right)^{j} \cos \left(L_{i} k_{n}^{(j)}+\gamma_{i}+\frac{\pi j}{2}\right)=0 .
$$

C помощью такого подхода можно немедленно показать, что количество $r$ вспомогательных сплетающих последовательностей конечно и определяется как минимальное целое положительное число, для которого выполнено условие

$$
\alpha_{r} \equiv \sum_{i=1}^{N_{\Gamma}}\left|a_{i}\left(\frac{L_{i}}{L_{0}}\right)^{r}\right|<1
$$

т.е. когда возможно обращение уравнения (24) по формуле (14). Так как $L_{i} / L_{0}<1$, из (25) очевидно, что каждый квантовый граф характеризуется определенным конечным индексом $r$ (степенью нерегулярности), ограничивающим число сплетающих последовательностей. Полученная таким способом оценка степени нерегулярности спектра завышена, т.е., вообще говоря, она больше числа "наиболее периодичных" вспомогательных последовательностей, полученных с помощью (20), (22), необходимых для сплетения $k_{n}^{(r+1)}$ с $k_{n}^{(0)}$.

\section{3. ЯВНЫЕ РАСПРЕДЕЛЕНИЯ ДЛЯ КВАНТОВЫХ УРОВНЕЙ}

Существование "сквозного" среднего и разделяющей последовательности (15) для регулярных графов позволяет напрямую найти явные разложения корней спектрального уравнения в ряд по замкнутым периодическим орбитам. Получающиеся выражения для спектральной величины $f_{n}^{(0)}$ (например, отклонения от среднего расстояния $\delta_{n}^{(0)}$ между двумя значениями $s_{n, m}^{(0)}=k_{n+m}^{(0)}-k_{n}^{(0)}$ и т.д.) имеют вид [9]-[12]

$$
f_{n}^{(0)}=\bar{f}_{n}^{(0)}-\sum_{p} C_{p}^{(0)} \cos \left(\omega_{p}^{(0)} n+\varphi_{p}^{(0)}\right),
$$

где частоты $\omega_{p}^{(0)}$ определяются оптическими длинами периодических орбит $L_{p}^{(0)}$, $\omega_{p}^{(0)}=\pi L_{p}^{(0)} / L_{0}$. В то время как первый член разложения (26) определяет среднее значение величины $f_{n}^{(0)}$, последующая сумма описывает флуктуации вокруг среднего. Каждая из частот $\omega_{p}^{(0)}$ представляет собой целочисленную комбинацию

$$
\omega_{p}^{(0)}=\pi\left(m_{p, 1}^{(0)} \Omega_{1}+m_{p, 2}^{(0)} \Omega_{2}+\cdots+m_{p, N_{\mathrm{P}}}^{(0)} \Omega_{N_{\mathrm{P}}}\right),
$$

где величины $\Omega_{i}$ выражаются через длины ребер графа, $\Omega_{i}=l_{i} / L_{0}$, а коэффициенты $m_{p, i}^{(0)}$ указывают, сколько раз данная орбита проходит вдоль ребра $l_{i}$. Можно определить порядок орбиты

$$
m_{p}^{(0)}=m_{p, 1}^{(0)}+m_{p, 2}^{(0)}+\cdots+m_{p, N_{\mathrm{P}}-1}^{(0)}
$$

как полное число актов рассеяния, претерпеваемых частицей при следовании вдоль пути $p$. Если в сумму (26) включаются только члены с конечным числом гармоник $p$, 
удовлетворяющих условию $m_{p}^{(0)}<m^{(0)}$ для некоторого фиксированного $m^{(0)}$, то результатом будет $m$-е приближение к точному значению $f_{n}^{(0)}$ [4], [12].

Поскольку числа $\Omega_{i}$ удовлетворяют условию

$$
\Omega_{1}+\Omega_{2}+\cdots+\Omega_{N_{P}}=1
$$

только $N_{\mathrm{P}}-1$ из них являются независимыми. Исключая с помощью (29) одну из величин $\Omega_{i}$ (для определенности будем считать, что $i=N_{\mathrm{P}}$ ), запишем выражение для флуктуирующей части в терминах оставшихся независимых величин $\Omega_{i}$,

$$
\begin{aligned}
f_{n}^{(0)}=- & \sum_{p} C_{p}^{(0)}(-1)^{m_{p, N_{\mathrm{P}}}^{(0)}} \sin \left(\pi \left(\left(m_{p, 1}^{(0)}-m_{p, N_{\mathrm{P}}}^{(0)}\right) \Omega_{1}+\right.\right. \\
& \left.\left.+\cdots+\left(m_{p, N_{\mathrm{P}-1}}^{(0)}-m_{p, N_{\mathrm{P}}}^{(0)}\right) \Omega_{N_{\mathrm{P}}-1}\right) n+\varphi_{p}^{(0)}\right) .
\end{aligned}
$$

В дальнейшем будем считать, что $\Omega_{1}, \ldots, \Omega_{N_{\mathrm{P}}-1}$ являются алгебраически независимыми иррациональными числами (случай общего положения). Назовем орбиту алгебраически простой, если целые коэффициенты $\widetilde{m}_{p, i}^{(0)}=m_{p, i}^{(0)}-m_{p, N_{\mathrm{P}}}^{(0)}$ в $(27)$ не имеют общих делителей, и будем обозначать такую орбиту индексом $p p$. Заметим, что, вообще говоря, динамически простые орбиты, соответствующие однократному прохождению вдоль некоторой последовательности ребер при движении частицы по графу, отличаются от алгебраически простых орбит. В дальнейшем под "простыми орбитами" будут подразумеваться именно алгебраически простые орбиты.

Для получения статистических свойств величин $f_{n}^{(0)}$ можно воспользоваться хорошо известным фактом, что для любого иррационального числа $\alpha$ последовательность остатков

$$
x_{n}=[\alpha n] \quad(\bmod 1)
$$

при $n=1,2, \ldots$ распределена равномерно на интервале [0,1] [38], [39]. Поскольку аргументы тригонометрических функций ряда (30) определены по модулю $2 \pi$, можно заключить, что в случае, когда величины $\Omega_{i}$ являются независимыми иррациональными числами, перебор значений $f_{n}^{(0)}$ дает последовательность, статистически эквивалентную ряду

$$
f_{x}^{(0)}=\bar{f}^{(0)}-\sum_{p} \widetilde{C}_{p}^{(0)} \sin \left(\widetilde{m}_{p}^{(0)} x+\varphi_{p}^{(0)}\right)
$$

в котором $x$ - это набор из $N_{\mathrm{P}}-1$ независимых равномерно распределенных случайных величин, а коэффициенты $\widetilde{C}_{p}^{(0)}$ изменены в соответствии с выражением (30) и включают в себя все гармоники с заданным набором коэффициентов $\widetilde{m}_{p}^{(0)}$ для данного $m$. В дальнейшем знак тильда над коэффициентами будем опускать.

Распределение значений $\delta f^{(0)}=\delta f_{x}^{(0)}$ в таком случае получается из выражения [35], [40]

$$
P_{f}^{(0)}=\int \delta\left(\delta f^{(0)}-\delta f_{x}^{(0)}\right) d x=\int e^{i k \delta f^{(0)}}\left(\int e^{i k \delta f_{x}^{(0)}} d x\right) d k,
$$


которое с учетом разложения (32) имеет вид

$$
P_{f}^{(0)}=\int d k e^{i k \delta f^{(0)}} \int_{0}^{2 \pi} \prod_{p} \Lambda_{p}^{(0)}(x) d x
$$

где $d x=\prod_{i=1}^{N_{\mathrm{P}}} d x_{i} /(2 \pi)$. Каждый множитель

$$
\Lambda_{p}^{(0)}(\mathbf{x})=\exp \left[i k C_{p}^{(0)} \sin \left(m_{p}^{(0)} x+\varphi_{p}^{(0)}\right)\right]
$$

определяет вклад соответствующей периодической орбиты $p$ в характеристическую функцию разложения (34). Учет в (34) гармоник, подчиняющихся условию $m_{p}^{(0)} \leqslant$ $m$, дает $m$-е приближение к функции распределения $P_{f}^{(0)}\left(\delta f^{(0)}\right)$ [35], [41].

Первоначально, поскольку все множители $\Lambda_{p}$ в $(34)$ зависят от одинакового набора переменных х, вклады разных орбит в функцию распределения сплетены. Заметим, однако, что при разложении конечных приближений (34) в ряд,

$$
I_{\{p\}}=\int_{0}^{2 \pi} \prod_{p} \Lambda_{p}^{(0)}(x) d x=\int_{0}^{2 \pi} \prod_{p}\left(\sum_{n_{p}=0}^{\infty} \frac{\left(i k C_{p}^{(0)}\right)^{n_{p}}}{n_{p} !} \cos ^{n_{p}}\left(\mathbf{m}_{p}^{(0)} \mathbf{x}+\varphi_{p}^{(0)}\right)\right) d x
$$

вклады от сплетений разных гармоник возникают благодаря "резонансам" между разными орбитами. Действительно, поскольку члены разложения в (32) взаимно ортогональны на интервале $[0,2 \pi]$ по каждой из переменных $x_{i}$, то основной вклад в произведение разложений от разных экспонент $\Lambda_{p}^{(0)}(\mathbf{x})$ вносят интегралы от степеней отдельных гармоник, в то время как большинство перекрестных членов, т.е. интегралов от произведения гармоник с разными $p$, обращаются в нуль. Разложения вида (36) можно рассматривать и с более общей алгебраической точки зрения, но необходимый результат получается из простой подстановки разложения

$$
\cos ^{n} x=\frac{2}{2^{n}} \sum_{k=0}^{\lfloor(n-1) / 2\rfloor} C_{n}^{k} \cos [(n-2 k) x]+h_{n},
$$

где $\lfloor\cdot\rfloor$ - наибольшее целое значение, $h_{n}$ - постоянная величина [42]. Понятно, что ненулевой вклад вносят только резонансные члены из (36), для которых выполняются условия вида

$$
\left(n_{1}-2 k_{1}\right) \mathbf{m}_{1}^{(0)} \pm\left(n_{2}-2 k_{2}\right) \mathbf{m}_{2}^{(0)} \pm \cdots \pm\left(n_{l}-2 k_{l}\right) \mathbf{m}_{l}^{(0)}=0 .
$$

Как показано в [9]-[12], амплитуда гармоники, соответствующей частоте (27) в ряде (26), пропорциональна произведению коэффициентов рассеяния на вершинах графа, поэтому вклад резонансных членов убывает в порядках малости соответствующих степеней $\left(C_{p_{1}}^{(0)}\right)^{n_{p_{1}}}\left(C_{p_{2}}^{(0)}\right)^{n_{p_{2}}} \ldots\left(C_{p_{l}}^{(0)}\right)^{n_{p_{l}}}$. Поскольку каждый из коэффициентов рассеяния по модулю меньше единицы, коэффициент $C_{p}^{(0)}$ экспоненциально убывает с ростом $m_{p}^{(0)}$, и соответственно амплитуды перекрестных членов убывают экспоненциально с ростом $m_{p}^{(0)} n_{p}$, где

$$
n_{p}=n_{p_{1}}+n_{p_{2}}+\cdots+n_{p_{l}} .
$$


Понятно также, что среди резонансных членов с данным $m_{p}^{(0)}$ наибольший вклад вносят перекрестные члены, соответствующие разным кратностям одной и той же простой орбиты $p p$, для которых $\mathbf{m}_{p_{1}}=\mathbf{m}_{p p} \nu_{1}, \mathbf{m}_{p_{2}}=\mathbf{m}_{p p} \nu_{2}, \ldots, \mathbf{m}_{p_{l}}=\mathbf{m}_{p p} \nu_{l}$,

$$
\left(n_{1}-2 k_{1}\right) \nu_{1} \pm\left(n_{2}-2 k_{2}\right) \nu_{2} \pm \cdots \pm\left(n_{l}-2 k_{l}\right) \nu_{l}=0
$$

поскольку для них условие (40) может выполняться, вообще говоря, для меньших $n_{p}$.

Воспользуемся этими рассуждениями, чтобы упростить интеграл для $P_{f}^{(0)}\left(\delta f^{(0)}\right)$. Например, в качестве простого физического приближения можно пренебречь в разложении (34) вкладами от резонансов между разными алгебраически простыми орбитами. Такое пренебрежение эквивалентно "расплетанию" множителей $\Lambda_{p}^{(0)}(x)$, соответствующих различным простым орбитам, т.е. введению независимого набора переменных $x_{p p}$ для каждой из простых орбит,

$$
\int \prod_{p} \Lambda_{p}^{(0)}(x) d x \rightarrow \prod_{p p} \int\left(\prod_{\nu_{p p}} \Lambda_{p p}^{(0)}\left(\nu_{p p} x_{p p}\right) d x_{p p}\right) .
$$

В таком случае вероятность распределения запишется в виде

$$
P_{f}^{(0)}\left(\delta f^{(0)}\right)=\int d k e^{i k \delta f^{(0)}} \prod_{p p} Q_{p p}^{(0)}\left(k C_{p p, \nu}^{(0)}\right),
$$

где каждый множитель

$$
Q_{p p}^{(0)}=\int_{0}^{2 \pi} \exp \left[i k \sum_{\nu} C_{p p, \nu}^{(0)} \cos \left(m_{p p}^{(0)} \nu x+\varphi_{p p, \nu}^{(0)}\right)\right] d x
$$

соответствует простой периодической орбите $p p$, и сумма по $\nu$ в (43) берется по кратностям повторения $p p$.

Более радикальное приближение возникает, если пренебречь резонансами между любыми несовпадающими орбитами, что эквивалентно полному "расплетанию" множителей $\Lambda_{p}^{(0)}(x)$ с различными $p$, т.е. введению независимых фаз $\theta_{p}$ для каждой из орбит,

$$
\begin{aligned}
& \int \Lambda_{p_{1}}^{(0)}(x) \Lambda_{p_{2}}^{(0)}(x) \ldots \Lambda_{p_{n}}^{(0)}(x) d x \rightarrow \\
& \quad \rightarrow \int \Lambda_{p_{1}}^{(0)}\left(\theta_{p_{1}}\right) \Lambda_{p_{2}}^{(0)}\left(\theta_{p_{2}}\right) \ldots \Lambda_{p_{n}}^{(0)}\left(\theta_{p_{n}}\right) d \theta_{p_{1}} d \theta_{p_{2}} \ldots d \theta_{p_{n}} .
\end{aligned}
$$

Поскольку величины $x_{i}$ равномерно распределены, распределение $P_{m_{p}^{(0)}}\left(\theta_{p}\right)$ для фаз $\theta_{p}=\mathbf{m}_{p}^{(0)} \mathbf{x}+\varphi_{p}^{(0)}$ на интервале длины $2 \pi m_{p}^{(0)}$ легко получить с помощью свертки $N_{\mathrm{P}}-1$ распределений для отдельных $x_{i}$,

$$
P_{f}^{(0)}\left(\delta f^{(0)}\right)=\int d k e^{i k \delta^{(0)}} \prod_{p} \int_{0}^{2 \pi m_{p}^{(0)}} \exp \left[i k C_{p}^{(0)} \sin \theta_{p}\right] P_{m_{p}^{(0)}}\left(\theta_{p}\right) d \theta_{p}
$$


Используя периодичность множителей $\Lambda_{p_{1}}(\theta)$ по $\theta$, получаем

$$
P_{f}^{(0)}\left(\delta f^{(0)}\right)=\int d k e^{i k \delta^{(0)}} \prod_{p} \int_{0}^{2 \pi} \exp \left[i k C_{p}^{(0)} \sin \varkappa_{p}\right] \widehat{P}\left(\varkappa_{p}\right) d \varkappa_{p},
$$

где

$$
\widehat{P}\left(\varkappa_{p}\right)=\sum_{n=0}^{m_{p}^{(0)}} P_{\theta_{p}}\left(\varkappa_{p}+2 \pi n\right) \sim \frac{1}{2 \pi}
$$

для больших $m_{p}^{(0)}$. В результате интеграл в (34) приводится к простому выражению

$$
P_{f}^{(0)}\left(\delta f^{(0)}\right)=\int d k e^{i k \delta^{(0)}} \prod_{p} J_{0}\left(k C_{p}^{(0)}\right),
$$

где $J_{0}(x)$ - функция Бесселя нулевого порядка [40]. Таким образом, в случае пренебрежения перекрестными членами в разложении (36) характеристическая функция распределения $P_{f}^{(0)}\left(\delta f^{(0)}\right)$ представляет собой произведение членов, соответствующих простым (42) или всем имеющимся (48) периодическим орбитам.

При необходимости вклад резонансных членов в характеристическую функцию может учитываться явно, хотя численный анализ показывает, что для общего описания форм спектральных распределений достаточно сложных квантовых графов, не имеющих специальных соотношений для длин ребер, это не является необходимым. Получение общего вида характеристической функции для $P_{f}^{(0)}\left(\delta f^{(0)}\right)$ в виде произведения по периодическим орбитам (48) представляет собой задачу той же алгоритмической сложности, что и построение суммы Гутцвиллера (3) для нахождения спектральной плотности.

Распределения вида (48) часто встречаются в различных теориях и приложениях, где используются модели случайных сигналов [43], [44]. Например, такие распределения возникают при анализе интенсивности интерферирующих телекоммуникационных сигналов, в теории коммуникаций [45], инженерии [46], при описании распространений волн в случайных средах, биофизике [47] и других областях. Таким образом, в приближении случайных фаз отдельные орбиты выступают в качестве отдельных “каналов" в фазовом пространстве.

Многие свойства распределений (48) для семейств огибающих суммы случайных сигналов доступны для прямых исследований и хорошо изучены аналитически. Например, можно проследить, как из сумм вида (32) могут возникать различные универсальные предельные распределения, в частности распределения Гаусса, Накагами-Райса и др. Заметим, что в приближении статистически независимых вкладов от разных периодических орбит обращение к предельным теоремам физически обоснованно в силу экспоненциального убывания величин коэффициентов $C_{p}^{(0)}$ и экспоненциального увеличения их числа с ростом $m_{p}^{(0)}$, в результате чего величина статистического отклонения $\sigma_{i}$ общего члена ряда мала по сравнению со статистическим отклонением всей суммы (теорема Линдеберга-Феллера [48]).

Как показывают численные расчеты, распределение флуктуаций $\delta_{n}$, описываемых суммой (26), для достаточно сложных графов (например, для шестивершинной 
гидры или полностью связного тетраэдра) хорошо приближается распределением Гаусса со среднеквадратичным отклонением [35], [41], [49], [50]

$$
\Delta^{2}=\frac{1}{2} \sum_{p}\left(C_{p}^{(0)}\right)^{2}
$$

Точно такое же распределение с тем же среднеквадратичным отклонением (49) возникает в приближении статистически независимых вкладов от разных периодических орбит из закона нормального распределения для суммы случайных величин $X_{i}$ со статистическими отклонениями $\sigma_{i}^{2}$,

$$
\Delta^{2}=\left\langle\left(\delta f^{(0)}\right)^{2}\right\rangle=\sum_{p}\left(C_{p}^{(0)}\right)^{2}\left\langle\cos ^{2} x_{p}\right\rangle=\frac{1}{2} \sum_{p}\left(C_{p}^{(0)}\right)^{2} .
$$

Таким образом, несмотря на то что в результате пренебрежения резонансными вкладами и введения независимых фаз разложение (26) перестает, вообще говоря, сходиться к корням спектрального уравнения, такое приближение сохраняет общие свойства распределения $P_{f}^{(0)}\left(\delta f^{(0)}\right)$.

Изложенный выше метод применим для описания распределений других спектральных характеристик (огибающих сумм случайных сигналов). Например, для разности значений $s_{m, n}^{(0)}=k_{n+m}-k_{n}$ имеем гармонический ряд

$$
s_{m, n}^{(0)}=\frac{\pi}{L_{0}} m-\sum_{p} D_{p, m}^{(0)} \cos \left(\omega_{p}^{(0)} n+\omega_{p}^{(0)} \frac{m}{2}\right),
$$

где

$$
D_{p, m}^{(0)}=\frac{4}{L_{0}} \frac{A_{p}^{(0)}}{\omega_{p}^{(0)}} \sin \left(\frac{\omega_{p}^{(0)}}{2}\right) \sin \left(\frac{\omega_{p}^{(0)} m}{2}\right) .
$$

Согласно предыдущим рассуждениям эта последовательность описывается случайным рядом

$$
s_{m, x}^{(0)}=\frac{\pi m}{L_{0}}-\sum_{p} D_{p, m}^{(0)} \cos \left(\mathbf{m}_{p}^{(0)} \mathbf{x}+\frac{\omega_{p}^{(0)} m}{2}\right),
$$

значения которого распределены по формуле

$$
P_{s_{m}}^{(0)}\left(s_{m}^{(0)}\right)=\int d k e^{i k\left(s_{m}^{(0)}-\pi m / L_{0}\right)} F_{s_{m}}^{(0)}(k)
$$

Характеристическая функция $F_{s_{m}}^{(0)}(k)$ распределения $P_{s_{m}}^{(0)}\left(s_{m}^{(0)}\right)$ может быть найдена точно по формуле (34) или в приближении независимых простых орбит (43) описана произведением величин $Q_{p}^{(0)}\left(k D_{p, m}^{(0)}\right)$, получаемых из вида коэффициентов $D_{p, m}^{(0)}$ для каждого $p$. В приближении случайных вкладов функция $F_{s_{m}}^{(0)}(k)$ определяется произведением $J_{0}\left(k D_{p, m}^{(0)}\right)$ согласно $(48)$. В частности, для $m=1$ получаем описание статистики разницы между ближайшими соседями $s_{n}^{(0)}=k_{n}^{(0)}-k_{n-1}^{(0)}$.

Такая же форма распределения вероятности может быть найдена для величины $\xi_{n}^{(0)}=\left(\delta_{n}^{(0)}+\delta_{n-1}^{(0)}\right) / 2$, используемой в $(23)$ и имеющей разложение вида $(26)$ с коэффициентами

$$
E_{p}^{(0)}=\frac{1}{\pi} \frac{A_{p}^{(0)}}{\omega_{p}^{(0)}} \sin \omega_{p}^{(0)}
$$


и фазами $\varphi_{p}=\left(\pi-\omega_{p}^{(0)}\right) / 2$, для флуктуаций энергии $\delta E_{n}=k_{n}^{2}-\bar{k}_{n}^{2}=\frac{\pi^{2}}{L_{0}^{2}}\left(\delta_{n}^{(0)}\right)^{2}$, разлагающихся в ряд с коэффициентами

$$
F_{p}^{(0)}=\frac{2}{\pi} \frac{A_{p}^{(0)}}{\omega_{p}^{(0)}}\left(\frac{2}{\omega_{p}^{(0)}} \sin \left(\frac{\omega_{p}^{(0)}}{2}\right) \cos \left(\frac{\omega_{p}^{(0)}}{2}\right)\right)
$$

и фазой $\varphi_{p}=0$, и т.д. Используя распределения этих величин, можно описать статистику более сложных объектов, например автокорреляционной функции $R_{2}(x)$, определяемой в терминах $k_{n}$ как

$$
R_{2}(x)=\frac{\pi}{L_{0}} \lim _{N \rightarrow \infty} \frac{1}{N} \sum_{n=1}^{N} \sum_{m \neq 0} \delta\left(k_{n+m}-k_{n}+x\right) .
$$

Формфактор $K_{2}(\tau)$ записывается в виде

$$
K_{2}(\tau)=\frac{\pi}{L_{0}} \lim _{N \rightarrow \infty} \frac{1}{N} \sum_{n=1}^{N} \sum_{m=1}^{N} e^{-i\left(k_{n+m}-k_{n}\right) \tau}=\frac{\pi}{L_{0}}\left\langle\sum_{m} e^{-i\left(k_{n+m}-k_{n}\right) \tau}\right\rangle_{n} .
$$

Для регулярных квантовых графов со спектральными разложениями вида (26) усреднение по $n$ в (58) может быть выполнено по случайной переменной (53)

$$
K_{2}(\tau)=\frac{\pi}{L_{0}} \sum_{m}\left\langle e^{-i s_{m} \tau}\right\rangle_{s_{m}}=\frac{\pi}{L_{0}} \sum_{m=1}^{\infty} e^{-i \frac{\pi m}{L_{0}} \tau} F_{s_{m}}^{(0)}(k)
$$

для $\tau \neq 0$, и, таким образом,

$$
R_{2}(x)=\frac{\pi}{L_{0}} \sum_{m=1}^{\infty} P_{s_{m}}^{(0)}(x) .
$$

Подчеркнем, что полученные формулы для распределений вероятности представляют собой замкнутые выражения, последовательно описывающие спектральные характеристики в терминах теории периодических орбит. Отдельные примеры аналитических выражений для распределений вероятности спектральных величин были получены ранее (см., например, [51]) для некоторых простых графов на основе прямого анализа свойств соответствующих спектральных уравнений. Благодаря наличию явных спектральных разложений (26) изложенный выше метод позволяет исследовать подобные выражения систематически, в общем контексте теории периодических орбит.

В силу приведенных выше рассуждений для получения приближенных выражений для характеристических функций каждой из спектральных величин можно пользоваться разложениями вида (48) и (42).

\section{4. СПЕКТРАЛЬНАЯ ИЕРАРХИЯ}

Для квантовых графов общего положения условие (13) не выполняется. Для таких систем можно предложить более общую схему решения, основанную на использовании вспомогательной системы разделяющих последовательностей (17). Суть 
подхода состоит в связывании соседних последовательностей в иерархии посредством соотношения

$$
k_{n}^{(j-1)}=\int_{k_{n-1}^{(j)}}^{k_{n}^{(j)}} k d N^{(j-1)},
$$

где $N^{(j-1)}(k)$ представляет собой спектральную лестницу для $k_{n}^{(j-1)}$. Каждая спектральная лестница $N^{(j)}(k)$ может быть разложена на среднюю (монотонную) и осциллирующую части, $N^{(j)}(k)=\bar{N}^{(j)}(k)+\delta N^{(j)}(k)$, где для каждого $j$

$$
\bar{N}^{(j)}(k)=\frac{L_{0}}{\pi} k-\frac{1}{2}=\bar{N}(k) .
$$

То, что последовательность $k_{n}^{(j-1)}$ сплетена (см. (17)) с последовательностью $k_{n}^{(j)}$ (или $N^{(j-1)}(k)$ с $N^{(j)}(k)$, см. рис. 5), означает, что $N^{(j-1)}\left(k_{n}^{(j)}\right)=n$. Тогда, используя (62), (10) и $k_{n}^{(j)}$ в виде (19), получим выражение

$$
\begin{aligned}
k_{n}^{(j-1)}=\frac{\pi n}{L_{0}} & +\frac{1}{2} \frac{\pi}{L_{0}}\left(\delta_{n}^{(j)}-\delta_{n-1}^{(j)}\right)- \\
& -\frac{1}{2} \frac{\pi}{L_{0}}\left(\left(\delta_{n}^{(j)}\right)^{2}-\left(\delta_{n-1}^{(j)}\right)^{2}\right)-\int_{k_{n-1}^{(j)}}^{k_{n}^{(j)}} \delta N^{(j-1)}(k) d k
\end{aligned}
$$

Если известно явное гармоническое разложение для $\delta N^{(j)}(k)(10)$,

$$
\delta N^{(j)}(k)=\frac{1}{\pi} \operatorname{Im} \sum_{p} A_{p}^{(j)} e^{i L_{p} k}
$$

то интеграл в (63) можно вычислить явно (в случае скейлинговых потенциалов величины $A_{p}^{(j)}$ не зависят от $k$ ) и получить для осциллирующей части $k_{n}^{(j-1)}$

$$
\delta_{n}^{(j-1)}=\bar{f}_{\delta}^{(j-1)}\left(\delta_{n}^{(j)}, \delta_{n-1}^{(j)}\right)-\sum_{p} C_{p}^{(j-1)} \sin \left(\omega_{p}^{(j-1)} n+\varphi_{p}^{(j-1)}\right),
$$

где

$$
\begin{aligned}
\bar{f}_{\delta}^{(j-1)}\left(\delta_{n}^{(j)}, \delta_{n-1}^{(j)}\right) & =\frac{1}{2}\left(\delta_{n}^{(j)}-\delta_{n-1}^{(j)}\right)-\frac{1}{2}\left(\left(\delta_{n}^{(j)}\right)^{2}-\left(\delta_{n-1}^{(j)}\right)^{2}\right), \\
C_{p}^{(j-1)} & =\frac{2}{L_{0}} \frac{A_{p}^{(j-1)}}{\omega_{p}^{(j-1)}} \sin \left(\frac{\omega_{p}^{(j-1)}}{2}\right)\left(\delta_{n}^{(j)}-\delta_{n-1}^{(j)}+1\right), \\
\varphi_{p}^{(j-1)} & =\omega_{p}^{(j-1)} \frac{\delta_{n}^{(j)}+\delta_{n-1}^{(j)}-1}{2} .
\end{aligned}
$$

В частном случае, когда $r=0, \delta_{n}^{(1)}=\delta_{n-1}^{(1)}=1 / 2$, соотношение (65) сводится к $(26)$. Видно, что величины $\delta_{n}^{(j-1)}$ на $(j-1)$-м уровне определяются через значения $\delta_{n}^{(j)}$ на предыдущем уровне иерархии.

Таким образом, удается получить явное описание перехода от одной разделяющей последовательности к другой. Заметим также, что если использовать систему разделителей на уровне $j$ в виде (20), явно заданную через спектральные значения на том же уровне, то выражение (65) можно формально рассматривать вне связи 
с остальными разделяющими последовательностями. Действительно, если значения коэффициентов $0<\alpha_{n}^{(j)}<1$ выбраны произвольно, то (65) будет формально являться самозамкнутым соотношением, определяющим величины $\delta_{n}^{(j)}$ в терминах “собственных" величин $\alpha_{n}^{(j)}, A_{p}^{(j)}$ и $\omega_{p}^{(j)}$. Например, в простейшем случае, когда в качестве разделителей на уровне $j$ используются серединные точки $(23)$ между $k_{n}^{(j)}$ и $k_{n+1}^{(j)}$, соотношение (65) запишется в виде

$$
\begin{aligned}
\delta_{n}^{(j)}=\frac{1}{2} & \left(\delta_{n+1}^{(j)}-\delta_{n-1}^{(j)}+2\right)\left(1-2\left(\delta_{n}^{(j)}+\frac{\delta_{n-1}^{(j)}+\delta_{n+1}^{(j)}}{2}\right)\right)- \\
& -\frac{2}{L_{0}} \sum_{p} \frac{A_{p}^{(j)}}{\omega_{p}^{(j)}} \sin \omega_{p}^{(j)}\left(n+\frac{1}{2}\left(\delta_{n}^{(j)}+\frac{\delta_{n+1}^{(j)}+\delta_{n-1}^{(j)}}{2}\right)\right) \times \\
& \times \sin \left(\frac{\omega_{p}^{(j)}}{2}\right)\left(1+\frac{\delta_{n+1}^{(j)}-\delta_{n-1}^{(j)}}{2}\right) .
\end{aligned}
$$

Нетривиальным следствием такой записи является явное выражение того, что значение каждой величины $\delta_{n}^{(j)}$ определяется значениями ее непосредственных соседей $\delta_{n-1}^{(j)}$ и $\delta_{n+1}^{(j)}$, а также набором параметров, характеризующих динамику движения на графе, входящих в разложение (65) через коэффициенты $A_{p}^{(j)}$ и $\omega_{p}^{(j)}$. Таким образом, зная значения соседних уровней справа и слева, можно точно восстанавливать значения серединных спектральных точек. Поэтому можно говорить о том, что структура физического спектра (а также вспомогательных последовательностей на других уровнях иерархии) определяется классической динамической информацией не только глобально, как в формуле Гутцвиллера, но и локально, что, например, позволяет восстанавливать отдельные недостающие спектральные значения.

Формула (65), описывающая переход разделяющих последовательностей между уровнями иерархии, обладает рядом интересных свойств, нетривиальным образом зависящих от вида коэффициентов $A_{p}^{(j)}$ и $\omega_{p}^{(j)}$. Свойства соотношений вида (65) и (67) представляют независимый интерес, который выходит за рамки данного обсуждения.

В дальнейшем соотношения вида (65) будут использованы для установления статистических свойств и распределений вероятностей величин $\delta_{n}^{(j)}$ на каждом уровне иерархии. Аналогичные разложения легко получить для других спектральных величин. Например, для разницы между $m$-ми соседями, $s_{n, m}^{(j-1)}=k_{n+m}^{(j-1)}-k_{n}^{(j-1)}$, разложение имеет вид

$$
\begin{aligned}
s_{n, m}^{(j-1)}=\frac{\pi m}{L_{0}} & +\bar{f}_{s}^{(j-1)}\left(s_{n-1, m}^{(j)}, s_{n, m}^{(j)}, s_{n, m-1}^{(j)}, \xi_{n}^{(j)}\right)+ \\
& +\frac{2}{L_{0}} \sum_{p} D_{p, m}^{(j-1)} \cos \left(\omega_{p}^{(j-1)} n-\frac{m}{2} \varphi_{p}^{(j-1)}\right),
\end{aligned}
$$

где коэффициенты разложения задаются в виде

$$
D_{p, m}^{(j-1)}=\frac{4}{L_{0}} \frac{A_{p}^{(j-1)}}{\omega_{p}^{(j-1)}}\left|d_{p, m}^{(j-1)}\right|
$$


с функцией

$$
\begin{gathered}
d_{p, m}^{(j-1)}=e^{i \omega_{p}^{(j-1)}\left(\xi_{n+m}^{(j)}+2 m\right) / 2} \sin \left(\frac{\omega_{p}^{(j-1)}}{2}\right)\left(\delta_{n+m}^{(j)}-\delta_{n+m-1}^{(j)}+1\right)- \\
-e^{i \omega_{p}^{(j-1)} \xi_{n}^{(j)} / 2} \sin \left(\frac{\omega_{p}^{(j-1)}}{2}\right)\left(\delta_{n}^{(j)}-\delta_{n-1}^{(j)}+1\right)
\end{gathered}
$$

и

$$
\begin{aligned}
\bar{f}_{s}^{(j-1)}= & \frac{1}{2}\left(s_{n-1, m}^{(j)}-2 s_{n, m}^{(j)}+s_{n, m-1}^{(j)}\right) \xi_{n}^{(j)}- \\
& -\frac{1}{2}\left(s_{n, m}^{(j)}-s_{n, m-1}^{(j)}-1\right)\left(s_{n, m}^{(j)}+s_{n-1, m}^{(j)}\right) .
\end{aligned}
$$

Примечательно, что зависимость от $\xi_{n}^{(j)}$ присутствует только в фазе $\varphi_{n}^{(j)}$, в то время как абсолютная величина $\left|d_{p, m}^{(j)}\right|$ зависит только от $s_{n, m}^{(j)}, s_{n, m \pm 1}^{(j)}$ :

$$
\begin{aligned}
2\left|d_{p, m}^{(j-1)}\right|^{2}=2 & -\cos \omega_{p}^{(j-1)} s_{n, m}^{(j)}+\cos \omega_{p}^{(j-1)} s_{n, m-1}^{(j)}+ \\
& +\cos \omega_{p}^{(j-1)} s_{n-1, m+1}^{(j)}-\cos \omega_{p}^{(j-1)}\left(s_{n-1, m}^{(j)}\right)- \\
& -\cos \omega_{p}^{(j-1)}\left(s_{n, m}^{(j)}-s_{n, m-1}^{(j)}\right)-\cos \omega_{p}^{(j-1)}\left(s_{n-1, m}^{(j)}-s_{n-1, m-1}^{(j)}\right) .
\end{aligned}
$$

Это несколько упрощает форму распределения значений $s_{m}^{(j-1)}$. Заметим также, что в силу соотношения $s_{n, m}^{(j)}-s_{n, m-1}^{(j)}+s_{n-1, m}^{(j)}=s_{n-1, m+1}^{(j)}$ только три из четырех величин в (72) являются независимыми. В частном случае $m=1$ соотношение (68) описывает развитие величин интервалов между ближайшими соседями $s_{n}^{(j-1)}=k_{n+1}^{(j-1)}-k_{n}^{(j-1)}$ вдоль иерархии.

\section{5. СТАТИСТИЧЕСКОЕ ОПИСАНИЕ СПЕКТРАЛЬНОЙ ИЕРАРХИИ}

Статистические свойства разделяющих последовательностей можно описать, используя связь разложений (63) на разных уровнях спектральной иерархии. Как и раньше, перебор значений индексов $n$ в аргументах гармонических функций (65) приводит к появлению случайных величин $x_{i}=\left(\Omega_{i} n\right)(\bmod 2 \pi)$ на каждом уровне $j$. Например, для отклонений от среднего имеем

$$
\delta_{x}^{(j-1)}=\bar{f}_{\delta}^{(j-1)}\left(\delta_{1, x}^{(j)}, \delta_{2, x}^{(j)}\right) \sum_{p} C_{p}^{(j-1)}\left(\delta_{x}^{(j)}\right) \cos \left(\mathbf{m}_{p}^{(j)} \mathbf{x}+\varphi_{p}\left(\delta_{x}^{(j)}\right)\right),
$$

где $\delta_{n}^{(j)} \rightarrow \delta_{1}^{(j)}(x)$ и $\delta_{n-1}^{(j)} \rightarrow \delta_{2}^{(j)}(x)$. Таким образом, в отличие от ряда $(32)$, стохастичность величин $\delta_{x}^{(j-1)}$ возникает не только из-за гармонических осцилляций на уровне $j-1$, но и из-за зависимости коэффициентов (73) от случайных последовательностей $\delta_{x}^{(j)}$ на предыдущем уровне иерархии. Форма распределения имеет вид

$$
\begin{aligned}
P_{\delta}^{(j-1)}\left(\delta^{(j-1)}\right)=\int & \delta\left(\delta^{(j-1)}-\delta^{(j-1)}\left(\delta_{x}^{(j)}, x\right)\right) \prod_{i=1}^{2} \delta\left(\delta_{i}^{(j)}-\delta_{i}^{(j)}\left(\delta_{x}^{(j+1)}, x\right)\right) \times \cdots \\
& \cdots \times \prod_{i=1}^{2} \delta\left(\delta_{i}^{(r)}-\delta_{i}^{(r)}(x)\right) \delta\left(\delta_{i}^{(r)}-\delta_{i}^{(r)}(x)\right) d x,
\end{aligned}
$$




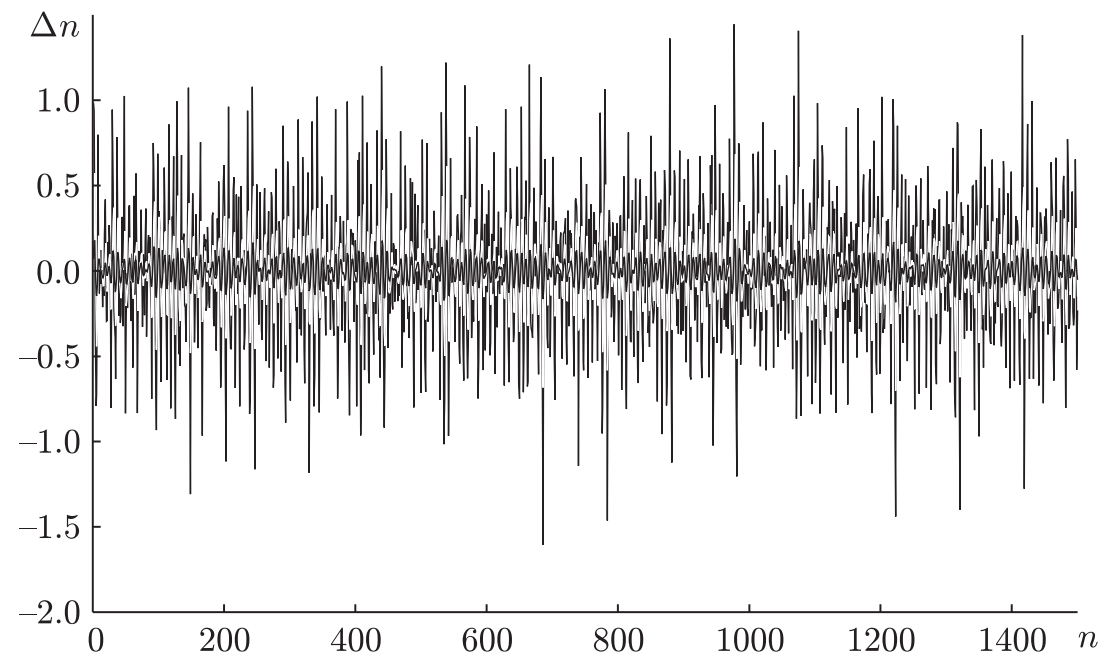

Рис. 6. Вид осцилляций $\delta_{n}^{(0)}, \delta_{n}^{(2)}$ и $\delta_{n}^{(5)}$ для разделительных последовательностей, полученных из решений уравнения (24) для шестивершинной гидры степени нерегулярности 5.

где произведение по $i=1,2$ в плотности для $\delta_{x}^{(j-1)}$ берется по числу прямых вкладов с предыдущего уровня $\delta_{x}^{(j)}$. В дальнейшем в выражениях вида (74) произведение по индексу $i$ будем опускать.

Для того чтобы упростить выражение (74), рассмотрим распределение вероятности на уровне $r-1$,

$$
P_{\delta}^{(r-1)}=\int \delta\left(\delta^{(r-1)}-\delta^{(r-1)}\left(\delta^{(r)}, x\right)\right) \delta\left(\delta^{(r)}-\delta^{(r)}(x)\right) d x d \delta^{(r)} .
$$

Разбивая область определения $x$ на области монотонности функции $\delta(x)$ и выражая плотность вероятности нахождения значений $x$, приводящих к данному значению $\delta$, перепишем условное распределение $P_{\delta}^{(r)}\left(\delta_{x}^{(r)} \mid x\right)$ через $P_{\delta}^{(r)}\left(x \mid \delta^{(r)}\right)$ (теорема Байеса)

$$
P_{\delta}^{(r-1)}=\int \delta\left(\delta^{(r-1)}-\delta^{(r-1)}\left(\delta^{(r)}, x\right)\right) P_{\delta}^{(r)}\left(x \mid \delta^{(r)}\right) P_{\delta}^{(r)}\left(\delta^{(r)}\right) d x d \delta^{(r)}
$$

где $P\left(\delta^{(r)}\right)$ - функция распределения величины $\delta^{(r)}$ на регулярном уровне иерархии.

Поскольку $\delta_{n}^{(j)}$ являются быстро осциллирующими функциями от $n$ (см. рис. 6$)$, обратная функция быстро покрывает область значений $x$. Кроме того, поскольку степень приближения $m^{(r)}$, используемая для вычисления $\delta^{(r)}$ на регулярном уровне, не зависит от степени приближения $m^{(r-1)}$ на уровне $r-1$ (на разных уровнях вычисляются разные многокомпонентные гармонические разложения), то в простейшем физическом приближении можно заменить $P^{(r)}(x \mid \delta) \sim P^{(r)}(x) \sim$ const, и тогда выражение (76) принимает вид

$$
P_{\delta}^{(r-1)}=\int \delta\left(\delta^{(r-1)}-\delta^{(r-1)}\left(\delta^{(r)}, x\right)\right) P\left(\delta^{(r)}\right) d x^{(r-1)} d \delta^{(r)},
$$


что соответствует введению независимого набора переменных $x$ на уровне $r-1$. В результате возникает удобное приближение, в котором $\delta^{(r)}$ и $x$ выступают в роли независимых случайных величин, по которым производится усреднение.

Такой подход можно обобщить на высшие уровни иерархии. Начиная с распределения $P_{f}^{(r)}$ на регулярном уровне, можно последовательно приближать форму распределений вероятности $P_{f}^{(j)}$ на каждом уровне $j$, включая последний, физический уровень. Ясно также, что, поскольку распределения на регулярном уровне $P_{f}^{(r)}$ получаются из одного интегрирования (61), они имеют ту же структуру, что и $P_{f}^{(0)}$ для регулярных графов, хотя коэффициенты разложения $A^{(r)}$ для нерегулярных графов будут отличаться от $A^{(0)}$ для $r \neq 0$.

Опишем для примера переход между последовательными уровнями иерархии для последовательности флуктуаций $\delta_{n}^{(j)}$. Будем рассматривать флуктуации $\delta_{n}^{(j)}$ и $\delta_{n-1}^{(j)}$ на $j$-м уровне, появляющиеся в выражении (65), как независимые случайные величины $\delta_{1}$ и $\delta_{2}$, распределенные по $P_{\delta}^{(j)}(\delta)$. Соответственно для плотности $P_{\delta}^{(j-1)}(\delta)$ можно написать

$$
\begin{aligned}
P_{\delta}^{(j-1)}(\delta)=\int & \delta\left(\delta \bar{f}_{\delta}^{(j-1)}\left(\delta_{1}, \delta_{2}\right)+\sum_{p} C_{p}^{(j-1)} \sin \left(m_{p}^{(j-1)} x+\varphi_{p}^{(j-1)}\right)\right) \times \\
& \times P_{\delta}^{(j)}\left(\delta_{1}\right) P_{\delta}^{(j)}\left(\delta_{2}\right) d \delta_{1} d \delta_{2} d x
\end{aligned}
$$

Разлагая дельта-функционал в интеграл Фурье

$$
\begin{aligned}
P_{\delta}^{(j-1)}(\delta)=\int & \exp \left[i k\left(\delta-\bar{f}_{\delta}^{(j-1)}+\sum_{p} C_{p}^{(j-1)} \sin \left(m_{p}^{(j-1)} x+\varphi_{p}^{(j-1)}\right)\right)\right] \times \\
& \times P_{\delta}^{(j)}\left(\delta_{1}\right) P_{\delta}^{(j)}\left(\delta_{2}\right) d \delta_{1} d \delta_{2} d x d k
\end{aligned}
$$

и интегрируя по $x_{p}$, получаем

$$
P_{\delta}^{(j-1)}(\delta)=\int d k e^{i k \delta}\left\langle F_{\delta}^{(j-1)}(k)\right\rangle_{\Omega^{(j)}}
$$

где величина $F_{\delta}^{(j-1)}(k)$ аналогична характеристической функции в (33) или $(34)$, а $\langle\cdot\rangle_{\Omega^{(j)}}$ обозначает усреднение по произведению с весом $\Omega^{(j)}(\eta, k)$,

$$
\Omega^{(j)}(\eta, k)=e^{i k f_{\delta}^{(j-1)}\left(\delta_{1}, \delta_{2}\right)} P_{\delta}^{(j)}\left(\delta_{1}\right) P_{\delta}^{(j)}\left(\delta_{2}\right) .
$$

Обратим внимание, что получение каждой из характеристических функций распределения $P_{\delta}^{(j-1)}(\delta)$ требует не только обобщения вида разложения для $\delta^{(j-1)}$, но и дополнительного усреднения по "беспорядку", привнесенному разделительными последовательностями с предыдущего уровня иерархии. Из (81) видно также, что функция $f_{\delta}^{(j-1)}\left(\delta_{1}, \delta_{2}\right)$ связывает вклады флуктуаций $\delta_{1}$ и $\delta_{2}$ с предыдущего уровня иерархии.

Для приближенного описания распределений $P_{\delta}^{(j-1)}(\delta)$ усреднение по осцилляциям $j$-го уровня в $(80)$ может производиться в приближении независимых простых 
орбит или в приближении независимых фаз, аналогично (48). В некоторых случаях это может приводить к дополнительным упрощениям. Например, исходя из записи (68) для $s_{n, m}^{(j)}$ в обозначениях $s_{1}=s_{n-1, m}^{(j)}, s_{2}=s_{n, m}^{(j)}, s_{3}=s_{n, m-1}^{(j)}$ имеем

$$
s_{m}^{(j-1)}=\frac{\pi m}{L_{0}}+f_{s}^{(j-1)}\left(s_{1}^{(j)}, s_{2}^{(j)}, s_{3}^{(j)}, \xi^{(j)}\right)+\sum_{p} D_{p, m}^{(j-1)} \cos \left(\omega_{p}^{(j-1)} n-\frac{m}{2} \varphi^{(j-1)}(\xi)\right)
$$

где $\varphi_{m}^{(j)}$ - комплексная фаза $d_{p, m}^{(j)}(70)$. Соответственно распределение $s_{m}^{(j-1)}$ дается выражением

$$
P_{m}^{(j-1)}(s)=\int e^{i k(s-m)}\left\langle F_{s_{m}}^{(j-1)}(k)\right\rangle_{\Phi^{(j)}} d k
$$

аналогично (80). Как было отмечено выше, величины $D_{p, m}^{(j-1)}$ не зависят от переменной $\xi^{(j)}$, поэтому в приближении случайных фаз, при усреднении по аргументам осциллирующих гармоник $j$-го уровня, зависимость от фазы $\varphi_{p}$ в выражении для $P_{\delta}^{(j-1)}(\delta)$ пропадает, и весовая функция $\Phi^{(j)}$ принимает вид

$$
\begin{gathered}
\Phi^{(j)}\left(s_{1}, s_{2}, s_{3}\right)=e^{i k\left(s_{2}-s_{3}-1\right)\left(s_{2}+s_{1}\right) / 2}\left(\int e^{-i k\left(s_{1}-2 s_{2}+s_{3}\right) \xi / 2} P_{\xi}^{(j)}(\xi) d \xi\right) \times \\
\times P_{m}^{(j)}\left(s_{1}\right) P_{m}^{(j)}\left(s_{2}\right) P_{m}^{(j)}\left(s_{3}\right) .
\end{gathered}
$$

С помощью этих выражений можно, например, проследить развитие распределений вигнеровского типа, используя разделительные последовательности (23), (20) с (22) или решения (24) (см. рис. 7). С помощью таких разложений для простых линейных или степенных функций от $k_{n}^{(j-1)}$ можно исследовать статистические свойства более сложных объектов, упомянутых в разделе 3 , например формфактора $K_{2}(\tau)$, различных корреляционных функций и других величин, вдоль иерархии. При этом вычисления и усреднения можно проводить или с помощью точного интегрирования, как в (34), или используя приближения, например приближение случайных фаз.

\section{6. ОБСУЖДДНИЕ}

Предложенный метод аналитического описания спектра состоит в последовательном выделении регулярной составляющей из разупорядоченной последовательности физических уровней $k_{n}=k_{n}^{(0)}$ путем ее сплетения с некоторой явно заданной регулярной последовательностью $k_{n}^{(r+1)}=k^{(r+1)}(n)$. В случае квантовых графов почти периодическая спектральная последовательность $k_{n}=k_{n}^{(0)}$ связывается с периодической последовательностью

$$
k_{n}^{(r+1)}=\frac{\pi}{L_{0}}\left(n+\frac{1}{2}\right)
$$

Для простейшего случая регулярных графов, описанных в разделах 2 и 3, такая связь непосредственна, поэтому регулярные спектры разупорядоченны только в пределах периодической последовательности (85). Для того чтобы охватить масштабы флуктуаций физического спектра в общем случае, необходимо ввести несколько 


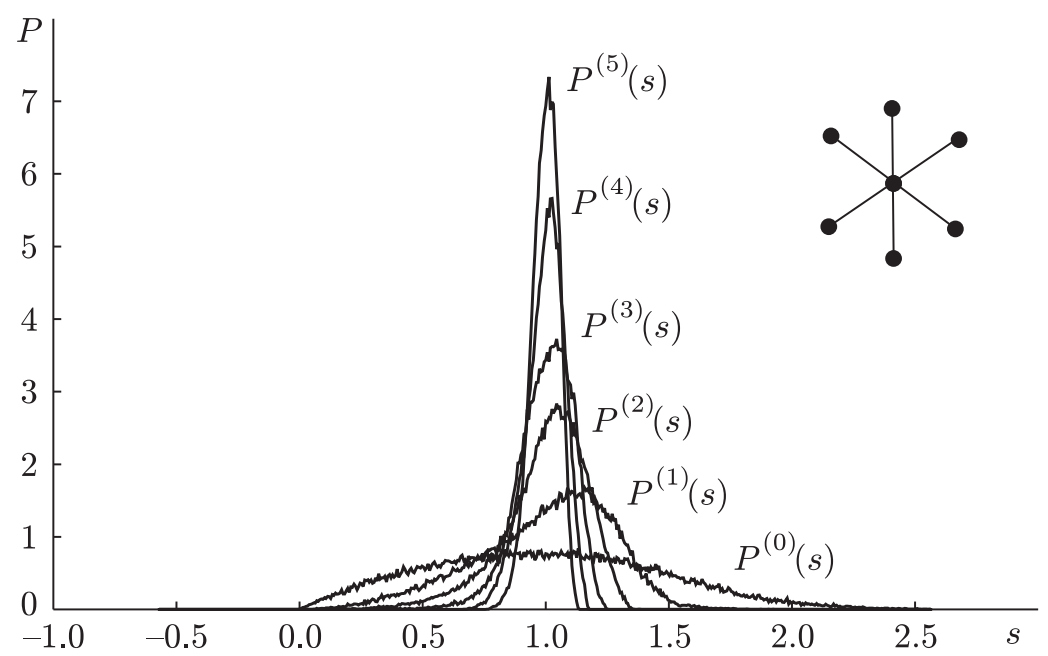

Рис. 7. Развитие формы распределения вероятности для расстояний между ближайшими соседями для случая шестивершинной гидры. Использованные здесь разделительные последовательности являются решениями уравнения (24). Распределение $P^{(0)}(s)$ на физическом уровне такое же, как на рис. 1. Спектральная лестница для регулярной последовательности $k_{n}^{(5)}$ показана на рис. 3.

дополнительных вспомогательных последовательностей, сплетающих $k_{n}^{(0)}$ с $k_{n}^{(r+1)}$. Минимальное число $r$ вспомогательных последовательностей $k_{n}^{(j)}$, необходимых для сплетения $k_{n}^{(r+1)}$ с $k_{n}^{(0)}$, выражает сложность спектральной задачи в контексте данного метода сплетения. Спектральная иерархия, таким образом, состоит из системы последовательностей $k_{n}^{(j)}, j=r+1, r, \ldots, 0$, каждая из которых структурирована предыдущей последовательностью в силу условия (17), а также соотношения (61) перехода от $k_{n}^{(j)}$ к $k_{n}^{(j-1)}$. Очевидно, что каждая из последовательностей иерархии $k_{n}^{(j)}$ разупорядоченна сильнее, чем структурирующая ее последовательность $k_{n}^{(j+1)}$, и в результате амплитуда флуктуаций на уровнях иерархии нарастает по мере убывания индекса $j$ от $j=r$ до 0 [13]-[15]. При этом индекс $n$ базовой последовательности снабжает иерархию глобально определенным квантовым числом $n$, которое с помощью соотношения (61) проносится с регулярного до нулевого (физического) уровня.

В результате применения гармонических разложений для спектральной лестницы возникают явные выражения для отдельных значений $k_{n}^{(0)}$, которые позволяют исследовать свойства спектра аналитически, в частности прослеживать развитие масштабов спектральных флуктуаций при переходе от менее разупорядоченных последовательностей к более разупорядоченным, а также исследовать возникновение универсальности в структуре спектральных распределений.

Хорошо известно, что распределения спектральных характеристик квантово-хаотических систем, в частности квантовых графов, обладают рядом универсальных свойств, диктуемых глобальными симметриями системы и описываемых тео- 


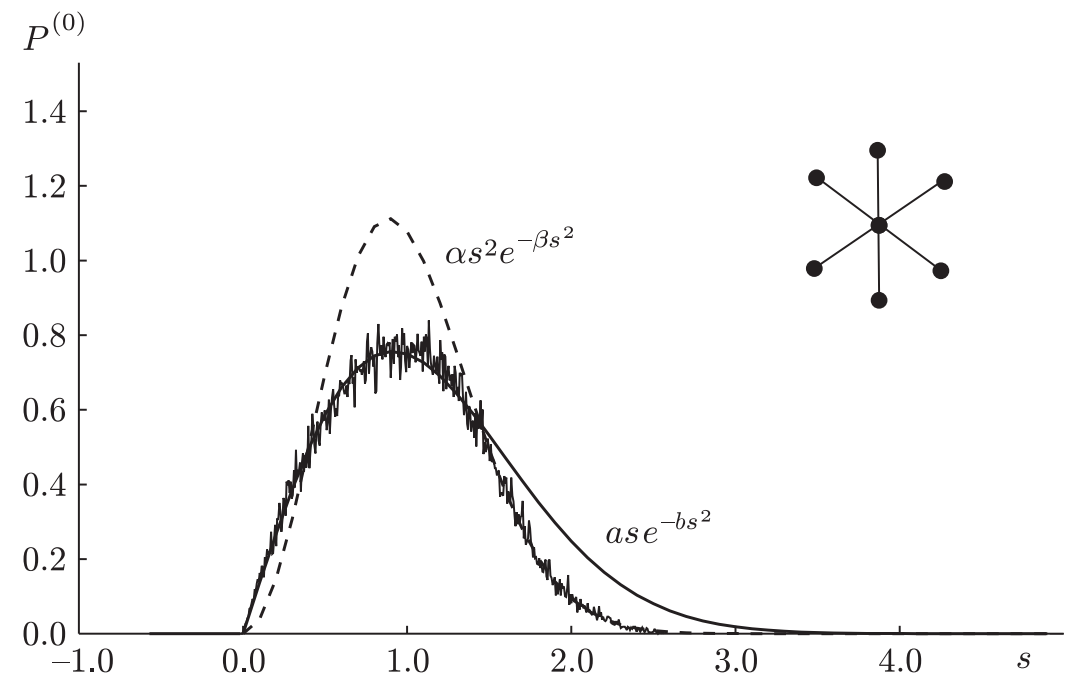

Рис. 8. Форма распределения вероятности для расстояний между ближайшими соседями для случая шестивершинной гидры, показанная на рис. 1, для малых $s$ приближается (ненормированной) функцией вида $a s e^{-b s^{2}}$, как в случае ортогонального гауссова ансамбля, в то время как хвост распределения хорошо приближается функцией вида $\alpha s^{2} e^{-\beta s^{2}}$, как в случае унитарного гауссова ансамбля.

рией случайных матриц (ТСМ) [2]-[4], [20], [49]-[51]. Однако, несмотря на то что универсальные функции распределения улавливают общие черты статистического поведения, они, вообще говоря, не являются точными для отдельно взятых систем. Например, в случае квантовых графов последовательности $k_{n}^{(j)}$ являются условно-периодическими, и поэтому расстояния между ближайшими соседями ограничены и не могут описываться распределениями с экспоненциальным хвостом, следующим из ТСМ [52], [53]. Функциональный вид распределений также может существенно отличаться от вида универсальных распределений, предсказанных ТСМ (см. рис. 8). Поэтому существенно, что предложенный выше подход, с одной стороны, позволяет описывать специфику той или иной системы, поскольку даже приближенные способы нахождения распределений вероятности через спектральные разложения, такие как приближение независимых простых орбит или приближение случайных фаз, содержат в себе специфическую информацию о конкретной системе. В частности, это позволяет исследовать величину статистического отклонения от предсказанного ТСМ универсального поведения. С другой стороны, этот общий подход может быть использован для аналитического исследования природы статистической универсальности.

Вопросы статистического описания тригонометрических разложений [54] часто рассматриваются в контексте теории слабо зависимых случайных величин (см., например, [55]-[57] и указанные там ссылки). Хотя методы и результаты этой теории 
плохо представлены в физической литературе, в ней имеется ряд строго доказанных аналитических результатов, непосредственно связанных с наблюдаемыми свойствами квантово-хаотических спектров.

В качестве примера можно привести утверждение о гауссовском характере распределения флуктуаций спектральной лестницы, $\delta N(k)=N(k)-\bar{N}(k)$, высказанное в качестве гипотезы о центральной предельной теореме для спектральных флуктуаций в [49], [50]. Эта гипотеза подтверждается численными исследованиями многих квантово-хаотических систем, в частности хаотических бильярдов. На основании анализа численно полученных спектров в [49], [50] было высказано предположение о том, что статистическое распределение значений $\delta N(k)$ является универсальным и имеет гауссовскую форму с вариацией, определяемую через спектральную жесткость,

$$
\Delta_{\infty}^{2}=\lim _{l \rightarrow \infty} \frac{1}{2 l} \int_{l}^{l}(\delta N(\varepsilon))^{2} d \varepsilon=\frac{1}{2} \sum_{p} A_{p}^{2}
$$

где $A_{p}$ - коэффициенты разложения в (3).

Аналогичная центральная предельная теорема хорошо известна в теории слабо зависимых случайных величин. В частности, в работах [58]-[69] было показано, что если последовательные частоты одномерных тригонометрических (или более общих [70], [71]) рядов

$$
f(x)=\sum_{k} c_{k} \cos \left(\omega_{k} x+\phi_{k}\right)
$$

возрастают достаточно быстро, то при некоторых дополнительных условиях вклады отдельных членов (или групп членов) в сумму ряда можно рассматривать как слабо зависимые или независимые случайные величины. При этом для них оказываются справедливы обобщения центральной предельной теоремы, устанавливающие нормальное распределение суммы ряда (87), аналогичные указанным в [49], [50],

$$
\frac{1}{\Delta_{K}} \sum_{k=1}^{K} c_{k} \cos \left(\omega_{k} x+\phi_{k}\right) \rightarrow N_{0,1},
$$

с вариацией

$$
\Delta_{K}^{2}=\frac{1}{2} \sum_{k=1}^{K} c_{k}^{2}
$$

Общее математическое описание распределений тригонометрических сумм довольно сложно и на данный момент не охватывает всех случаев обсуждаемых спектральных разложений. Например, для общих доказательств сходимости к предельному гауссовскому распределению [61]-[64] важную роль играет скорость роста частот ряда (87). В частности, в случае рядов с одной скалярной переменной $x$ для общего доказательства центральной предельной теоремы необходимо выполнение условия

$$
\frac{\omega_{k+1}}{\omega_{k}} \geqslant 1+\frac{\alpha_{k}}{\sqrt{k}}
$$


где $\alpha_{k} \rightarrow \infty$ [67], что означает экспоненциальную скорость роста частот, $\omega_{k} \sim e^{\sqrt{k}}$. Подобная скорость роста длин (простых) орбит, вообще говоря, не имеет места для случая графов или более сложных систем. Тем не менее нарушение условий вида (90) само по себе не означает, что сходимость к универсальному предельному распределению значений $f(x)$ отсутствует. Про сходимость распределений сумм рядов (87) к предельным распределениям при нарушении “критического" условия (90) известно достаточно мало, и еще меньше известно о поведении тригонометрических сумм от векторных аргументов, таких как (32) [72]-[77]. Тем не менее, как показано в [62], [71], [78], при определенных условиях сумма ряда (87) может сходиться к гауссовскому распределению при сколь угодно медленном возрастании частот $\omega_{k}$.

На основе результатов численных исследований спектров квантовых графов можно утверждать, что их общие статистические свойства описываются распределениями вероятности, обладающими характерными чертами универсальных распределений квантово-хаотических систем. Таким образом, на основе вышеприведенных аналитических результатов для квантовых графов можно предположить, что для сумм вида (32), (87) также справедливы обобщения центральной предельной теоремы (88) для тригонометрических сумм, с конечной (в силу ограниченности флуктуаций) вариацией

$$
\Delta^{2}=\left\langle\left(\delta f^{(0)}\right)^{2}\right\rangle=\sum_{p}\left(C_{p}^{(0)}\right)^{2}\left\langle\cos ^{2} x_{p}\right\rangle=\frac{1}{2} \sum_{p}\left(C_{p}^{(0)}\right)^{2} .
$$

Таким образом, возникает возможность аналитического выявления природы универсальности спектров квантово-хаотических систем, связывающего статистические (обычно феноменологические) теории с квазиклассической теорией периодических орбит.

Возможность получения явных тригонометрических разложений для функций от $k_{n}^{(j)}$ в случае квантовых графов позволяет существенно расширить круг спектральных характеристик, для которых может оказаться справедливой гипотеза, высказанная в [49], [50]. В качестве примера можно привести формы распределения для полусумм соседних флуктуаций $\xi_{n}^{(j)}=\left(\delta_{n}^{(j)}+\delta_{n-1}^{(j)}\right) / 2$ (см. рис. 9).

Важно также, что в дополнение к статистической универсальности, диктуемой поведением отдельных рядов (32), в рамках предложенного подхода имеется дополнительный механизм усреднения благодаря переходам между уровнями спектральной иерархии. Ясно, что высокая степень нерегулярности подразумевает соответствующее количество переходов $(61)$ и усреднений по случайным переменным $x_{p}^{(j)}$ и по разупорядоченным последовательностям разделителей $k_{n}^{(j-1)}$. В то время как распределения флуктуаций различных статистик на регулярном уровне хорошо приближаются гауссовским распределением, дополнительные усреднения по беспорядку могут приводить к отклонению от нормального закона, как, например, в случае распределения расстояний между ближайшими соседями $s_{n}=k_{n}-k_{n-1}$ (рис. 7), когда по мере приближения к физическому уровню возникает вигнеровская форма распределения вероятности. 


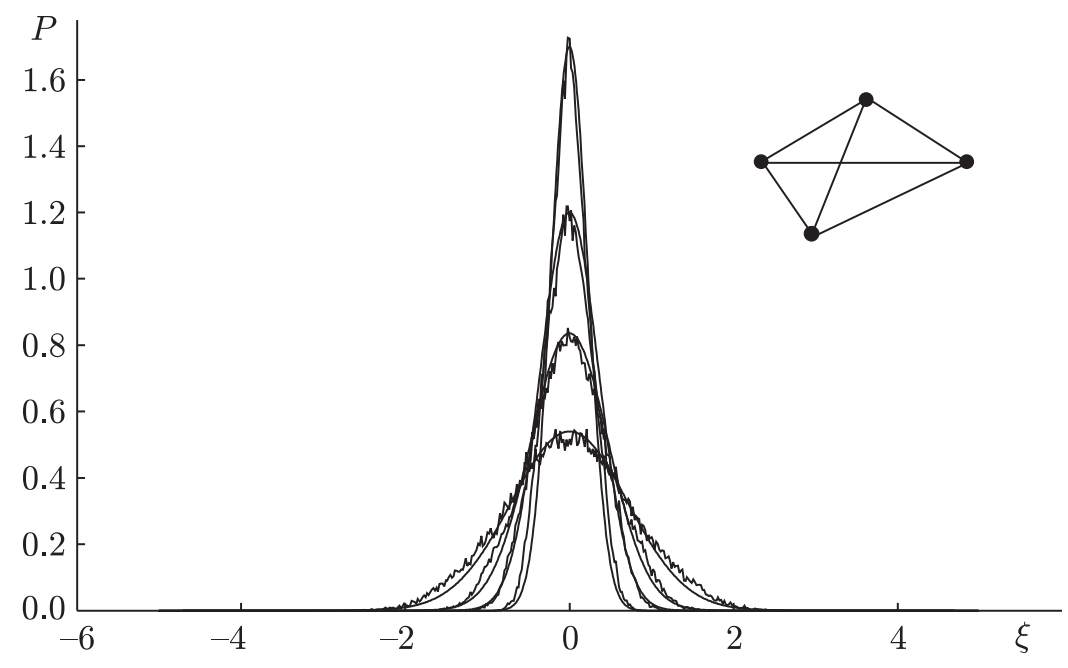

Рис. 9. Гистограммы 80000 значений полусумм соседних флуктуаций $\xi_{n}^{(j)}=\left(\delta_{n}^{(j)}+\delta_{n-1}^{(j)}\right) / 2$, полученных для полностью связного неоснащенного четырехвершинного графа. Сглаженные линии, аппроксимирующие гистограммы, соответствуют гауссовским распределениям.

Благодарности. Работа выполнена при частичной поддержке Sloan Schwartz Foundation.

\section{Список литературы}

[1] F. Barra, P. Gaspard, Phys. Rev. E, 63:6 (2001), 066215.

[2] H. Schanz, U. Smilansky, Phys. Rev. Lett., 84:7 (2000), 1427-1430.

[3] T. Kottos, U. Smilansky, Phys. Rev. Lett., 79:24 (1997), 4794-4797.

[4] T. Kottos, U. Smilansky, Ann. Phys., 274:1 (1999), 76-124.

[5] В.И. Арнольд, А. Авец, Эргодические проблемы классической механики, РХД, Ижевск, 1999.

[6] M. Gutzwiller, Chaos in Classical and Quantum Mechanics, Springer, New York, 1990.

[7] M.-J. Giannoni, A. Voros, J. Zinn-Justin (eds.), Chaos et physique quantique, Papers from the Fifty-second Session of the Summer School in Theor. Phys. (Les Houches, August 1-31, 1989), North-Holland, Amsterdam, 1991.

[8] Yu. Dabaghian, R. Jensen, R. Blümel, Phys. Rev. E, 63:6 (2001), 066201.

[9] Yu. Dabaghian, R. V. Jensen, R. Blümel, Писъма в ЖЭТФ, 74:4 (2001), 258-262.

[10] R. Blümel, Yu. Dabaghian, R. V. Jensen, Phys. Rev. Lett., 88:4 (2002), 044101.

[11] R. Blümel, Yu. Dabaghian, R. V. Jensen, Phys. Rev. E, 65:4 (2002), 046222.

[12] Yu. Dabaghian, R. V. Jensen, R. Blümel, ЖЖЭTФ, 121:6 (2002), 1399-1414.

[13] Yu. Dabaghian, R. Blümel, Phys. Rev. E, 68:5 (2003), 055201.

[14] Yu. Dabaghian, R. Blümel, Писъма в ЖЖЭТФ, 77:9 (2003), 530-533.

[15] Yu. Dabaghian, R. Blümel, Phys. Rev. E, 70:4 (2004), 046206.

[16] Б. М. Смирнов, УФН, 171:12 (2001), 1291-1315.

[17] R. Balian, C. Bloch, Ann. Phys., 60:2 (1970), 401-447; 64:1 (1971), 271-307; 69:1 (1972), $76-160$. 
[18] S. Gnutzmann, U. Smilansky, Adv. Phys., 55:5-6 (2006), 527-625.

[19] М. В. Фейгельман, В.В.Рязанов, В.Б. Тимофеев, УФН, 171:10 (2001), 1099-1115.

[20] O. Bohigas, M.-J. Giannoni, C. Schmidt, Phys. Rev. Lett., 52:1 (1984), 1-4.

[21] F. Haake, Quantum Signatures of Chaos, Springer, Berlin, 1992.

[22] M. L. Mehta, Random Matrices, 2 ed., Academic Press, Boston, MA, 1991.

[23] J.-P. Roth, "Le spectre du Laplacien sur un graphe", Thé orie du potentiel, Proc. Jacques Deny Colloquium (Orsay, France, June 20-23, 1983), Lecture Notes in Math., 1096, eds. G. Mokobodzki, D. Pinchon, Springer, Berlin, 1984, 521-539.

[24] K. G. Andersson, R. B. Melrose, Inv. Math., 41:3 (1977), 197-232.

[25] Г. М. Заславский, Н. Н. Филоненко, ЖЭТФ, 65:2 (1973), 643.

[26] Г. М. Заславский, УФН, 129:2 (1979), 211-238.

[27] N. Bohr, Phil. Mag., 26 (1913), 1-25; 476-502

[28] A. Sommerfeld, Ann. Phys., 51 (1916), 1-94; 356:18 (2006), 125-167.

[29] A. Einstein, "Zum Quantensatz von Sommerfeld und Epstein", Sammelband, 9-10, Deutsche Physikalische Gesellschaft, 1917, 82-92.

[30] L. Brillouin, J. Phys. Radium, 7 (1926), 353-368.

[31] J. B. Keller, Ann. Phys., 4:2 (1958), 180-188.

[32] E. Bogomolny, Nonlinearity, 5:4 (1992), 805-866.

[33] E. Bogomolny, Chaos, 2:1 (1992), 5-13.

[34] H. P. Baltes, E. R. Hilf, Spectra of Finite Systems, Bibliographisches Institut, Mannheim, 1976.

[35] Yu. Dabaghian, Phys. Rev. E, 75 (2007), 056214.

[36] Б. Я. Левин, Распределение корней целых функций, ГИТТЛ, М., 1956.

[37] E. Laguerre, Oeuvres de Laguerre publiées sous les auspices de l'Academie des sciences. T. I. Algèbre. Calcul intégral, eds. Ch. Hermite, H. Poincaré, E. Rouché, Gauthier-Villars, Paris, 1898; T. II. Géométrie, 1905.

[38] А.А. Карацуба, Основы аналитической теории чисел, Наука, М., 1983.

[39] Л. Кейперс, Г. Нидеррейтер, Равномерное распределение последовательностей, Наука, М., 1985.

[40] Ю. Дабагян, Писъма в ЖЭТФ, 83:12 (2006), 685-690.

[41] Yu. Dabaghian, Spectral statistics for scaling quantum graphs, arXiv: quant-ph/0608076, 2006.

[42] И.С. Градштейн, И.М. Рыжик, Таблицы интегралов, сумм, рядов и произведений, Физматлит, М., 1963.

[43] Б.Р. Левин (ред.), Статистическая теория связи и ее практическое применение, Радио и связь, М., 1979.

[44] M. Pätzold, Mobile Fading Channels: Modelling, Analysis and Simulation, MATLAB and Simulink Based Books, John Wiley and Sons, 2002.

[45] В. И. Тихонов, ВН. Харисов, Статистический анализ и синтез радиотехнических устройств и систем, Радио и связь, М., 1991.

[46] A. Abdi, H. Hashemi, S. Nader-Esfahani, IEEE Trans. Comm., 48:1 (2000), 7-12.

[47] D. Melkonian, T. Blumenthal, E. Gordon, Biol. Cybern., 81:5-6 (1999), 457-467.

[48] P. Beckmann, Probability in Communication Engineering, Harcourt, Brace and World, New York, 1967.

[49] R. Aurich, J. Bolte, F. Steiner, Phys. Rev. Lett., 73:10 (1994), 1356-1359.

[50] R. Aurich, A. Bäcker, F. Steiner, Internat. J. Modern Phys. B, 11:7 (1997), 805-849.

[51] F. Barra, P. Gaspard, J. Stat. Phys., 101:1-2 (2000), 283-319.

[52] Г. Бор, Почти периодические функиии, УРСC, М., 2005.

[53] C. Corduneanu, Almost Periodic Functions, Wiley, New York-London-Sydney, 1968.

[54] А. Зигмунд, Тригонометрические рядь, Мир, М., 1965. 
[55] Р. В. Гамкрелидзе, Ю.В. Прохоров, В.А. Статюлявичус (ред.), Теория вероятностей 6, Итоги науки и техники. Соврем. проблемы матем. Фундам. напр., 81, ВИНИТИ, М., 1991.

[56] Й. Сунклодас, “Аппроксимация распределений сумм слабо зависимых случайных величин нормальным распределением", Теория вероятностей 6, Итоги науки и техники. Соврем. проблемы матем. Фундам. напр., 81, ред. Р. В. Гамкрелидзе, Ю. В. Прохоров, В. А. Статюлявичус, ВИНИТИ, М., 1991, 140-199.

[57] P. Révész (ed.), Limit theorems in Probability and Statistics, 2nd Colloq. on Limit Theorems Probab. Theor. and Statist. (Veszprém, Hungary, June 21-26, 1982), Colloq. Math. Soc. János Bolyai, 36, North-Holland, Amsterdam, 1984.

[58] S. Takahashi, "Probability limit theorems for trigonometric series", Limit Theorems Probab. Theory (Keszthely, 1974), Colloq. Math. Soc. János Bolyai, 11, ed. P. Révész, North-Holland, Amsterdam, 1975, 381-397.

[59] W. Philipp, W.F. Stout, "Asymptotic fluctuation behavior of sums of weakly dependent random variables", Limit Theorems Probab. Theory (Keszthely, 1974), Colloq. Math. Soc. János Bolyai, 11, ed. P. Révész, North-Holland, Amsterdam, 1975, 273-296.

[60] W. Philipp, W. Stout, Almost Sure Invariance Principles for Partial Sums of Weakly Dependent Random Variables, Mem. Amer. Math. Soc., 161, AMS, Providence, RI, 1975.

[61] K. Fukuyama, S. Takahashi, Proc. Amer. Math. Soc., 127:2 (1999), 599-608.

[62] С. В. Левизов, Матем. заметки, 38:2 (1985), 242-247.

[63] K. Ôhashi, Anal. Math., 20:1 (1994), 11-25.

[64] J. Hawkes, Z. Wahrsch. Verw. Gebiete, 53:1 (1980), 21-33.

[65] R. Salem, A. Zygmund, Proc. Natl. Acad. Sci. USA, 33:11 (1947), 333-338; 34:2 (1948), $54-62$.

[66] R. Salem, A. Zygmund, Acta Math., 91:1 (1954), 245-301.

[67] P. Erdős, Magyar Tud. Acad. Mat. Kutat'o Int. Közl., 7 (1962), 37-42.

[68] A. Főldes, Studia Sci. Math. Hungar., 10:1-2 (1975), 141-146.

[69] W. Philipp, Acta Arith., 26:3 (1975), 241-251.

[70] I. Berkes, "On the asymptotic behaviour of $\sum f\left(n_{k} x\right)$ ", Limit Theorems Probab. Theory (Keszthely, 1974), Colloq. Math. Soc. János Bolyai, 11, ed. P. Révész, North-Holland, Amsterdam, 1975, 23-46.

[71] I. Berkes, Anal. Math., 4:3 (1978), 159-180.

[72] В. Ф. Гапошкин, УМН, 21:6(132) (1966), 3-82.

[73] В.Ф. Гапошкин, Матем. заметки, 16:6 (1974), 865-870.

[74] B. I. Golubov, J. Math. Sci., 24:6 (1984), 639-673.

[75] Б. И. Голубов, Докл. РАН, 20:5 (1979), 1103-1107.

[76] П. Л. Ульянов, УМН, 19:1(115) (1964), 3-69.

[77] K. Wang, Probab. Theory Related Fields, 98:2 (1994), 229-243.

[78] I. Berkes, Z. Wahrsch. Verw. Gebiete, 47:2 (1979), 157-161.

Поступила в редакцию 30.05.2007 